\title{
Neutrino mixing in SO(10) GUTs with a non-Abelian flavor symmetry in the hidden sector
}

\author{
Alexei Yu. Smirnov and Xun-Jie Xu \\ Max-Planck-Institut für Kernphysik, Postfach 103980, D-69029 Heidelberg, Germany
}

(Received 8 April 2018; published 21 May 2018)

\begin{abstract}
The relation between the mixing matrices of leptons and quarks, $U_{\mathrm{PMNS}} \approx V_{\mathrm{CKM}}^{\dagger} U_{0}$, where $U_{0}$ is a matrix of special forms [e.g., bimaximal (BM) and tribimaximal], can be a clue for understanding the lepton mixing and neutrino masses. It may imply the grand unification and the existence of a hidden sector with certain symmetry that generates $U_{0}$ and leads to the smallness of neutrino masses. We apply the residual symmetry approach to obtain $U_{0}$. The residual symmetries of both the visible and hidden sectors are $\mathbb{Z}_{2} \times \mathbb{Z}_{2}$. Their embedding in a unified flavor group is considered. We find that there are only several possible structures of $U_{0}$, including the BM mixing and matrices with elements determined by the golden ratio. Realization of the BM scenario based on the $\mathrm{SO}(10)$ grand unified theory with the $S_{4}$ flavor group is presented. Generic features of this scenario are discussed, in particular, the prediction of $C P$ phase $144^{\circ} \lesssim$ $\delta_{\mathrm{CP}} \lesssim 210^{\circ}$ in the minimal version.
\end{abstract}

DOI: 10.1103/PhysRevD.97.095030

\section{INTRODUCTION}

There is an appealing approximate relation between the mixing matrices of leptons, $U_{\mathrm{PMNS}}$, and quarks, $V_{\mathrm{CKM}}$ [1-5],

$$
U_{\mathrm{PMNS}} \simeq V_{\mathrm{CKM}}^{\dagger} U_{0},
$$

where $U_{0}$ is close to the bimaximal $(\mathrm{BM})\left(U_{\mathrm{BM}}\right)[6,7]$ or tribimaximal $(\mathrm{TBM})\left(U_{\mathrm{TBM}}\right)$ [8,9] mixing matrices. In particular, Eq. (1) gives a relation between the Cabibbo angle $\theta_{C}$ and the leptonic mixing angles $\theta_{13}$ and $\theta_{23}$ :

$$
\sin ^{2} \theta_{13} \simeq \sin ^{2} \theta_{23} \sin ^{2} \theta_{C} \text {. }
$$

The relation (1) is in a good agreement with available experimental results [10] and has been widely studied in the literature [11-26]. If not accidental, it can be the clue for understanding peculiar features of the lepton mixing and, eventually, the origins of neutrino masses. The following logical steps lead to a rather restricted scenario:

(1) The relation (1) implies that leptons "know" about quarks. It should be a kind of quark-lepton unification, probably the grand unification at highenergy scale. Indeed, the grand unification can

Published by the American Physical Society under the terms of the Creative Commons Attribution 4.0 International license. Further distribution of this work must maintain attribution to the author(s) and the published article's title, journal citation, and DOI. Funded by SCOAP ${ }^{3}$. ensure similarity of the Dirac mass matrices of quarks and leptons: $m_{D}^{\nu} \sim m_{D}^{\text {up }}$ and $m_{D}^{l} \sim m_{D}^{\text {down }}$. This leads to the appearance of mixing $\sim V_{\mathrm{CKM}}$ in the lepton sector. ${ }^{1}$

(2) At the same time, the difference between the quark and lepton mixing implies the existence of some new physics responsible for generation of matrix $U_{0}$. The structure of this matrix indicates certain underlying symmetry that is difficult to extend to the quark sector.

(3) It is natural to assume that the same new physics is responsible for $U_{0}$ and the smallness of neutrino masses. In the grand unification framework, the simplest way to get small neutrino masses is to invoke the high-mass scale type-I seesaw mechanism [27-31],

$$
m_{\nu}=-m_{D}^{\nu} \frac{1}{M_{R}} m_{D}^{\nu T},
$$

where $M_{R}$ is the mass matrix of right-handed (RH) neutrino components. Thus, in the seesaw mechanism, $M_{R}$ with specific properties could be responsible for the generation of $U_{0}$.

(4) The latter, however, implies a very strong (quadratic) hierarchy of masses of the neutrinos and enormous fine-tuning that is very difficult (if possible) to justify in the usual seesaw mechanism. One way

\footnotetext{
${ }^{1}$ Alternatively, the relations between the mass matrices can be obtained as a consequence of common flavor symmetry in both sectors.
} 
to solve this problem is to introduce the doubleseesaw mechanism in which the RH neutrinos themselves acquire masses via the seesaw mechanism [32]. The realization of the double-seesaw mechanism requires the introduction of new fermions $S$ with the Majorana mass matrix $M_{S}$ and Yukawa interactions with the RH neutrinos that lead to the Dirac mass matrix $M_{D}$. This opens up the possibility to cancel the strong hierarchy as a result of certain symmetry, which leads to the proportionality $m_{D} \propto M_{D}[33,34]$. Furthermore, the structure of the mass matrix of $S$, governed by certain symmetry, can eventually lead to the required mixing $U_{0}$.

These general arguments can be realized in the following scenario:

(i) There is the grand unification based on the $\mathrm{SO}(10)$ gauge symmetry group [35,36] with fermions in 16-plet representations that also include the $\mathrm{RH}$ neutrinos. This ensures similarity of the Dirac mass matrices of the quarks and leptons and also the coincidence of scales $M_{S N} \sim M_{\mathrm{GUT}}$.

(ii) A hidden sector that consists of singlet fermions and bosons of $\mathrm{SO}(10)$ exists. This sector couples with the visible one via the RH neutrino portal. The fermions $S$ that participate in the double-seesaw mechanism belong to this sector. Symmetries of the hidden sector lead to the matrix $U_{0}$ with the required properties.

(iii) Information about mixing in the hidden sector should be communicated to the visible sector. The minimal possibility is to fix the basis of states in all sectors (visible, portal, and hidden), and this can be done by introducing the basis-fixing symmetry [37-39]. In the case of three generations, the simplest possibility is the $\mathbb{Z}_{2} \times \mathbb{Z}_{2}$ symmetry [37]. In turn, such a symmetry can be a part of intrinsic symmetry of the theory, which is always present. The basisfixing symmetry ensures that all mass matrices of the visible sector and portal are diagonal. This symmetry is spontaneously broken in the hidden sector by interactions with flavons, leading to another unbroken (intrinsic) $\mathbb{Z}_{2} \times \mathbb{Z}_{2}$ and generating $U_{0}$. No such a structure exists in the quark sector.

(iv) Additional physics should be introduced to generate the Cabibbo-Kobayashi-Maskawa (CKM) mixing.

(v) In the visible sector, $\mathbb{Z}_{2} \times \mathbb{Z}_{2}$ is broken by another mechanism leading to the CKM mixing. The double-seesaw mechanism allows one to disentangle the generation of CKM and $U_{0}$ mixings. Furthermore, introducing the hidden sector allows one to construct economical renormalizable theory with flavor symmetry.

In general, $\mathbb{Z}_{2} \times \mathbb{Z}_{2}$ can lead to large mixing in $U_{0}$, but it does not produce specific structures such as BM or TBM.
To this end, non-Abelian symmetry should be introduced in the hidden sector. In other words, the basis symmetry $\mathbb{Z}_{2} \times \mathbb{Z}_{2}$ should be promoted to non-Abelian symmetry. In this paper, we study such a possibility. We focus on the symmetry issues: the interplay between the gauge $\mathrm{SO}(10)$ and discrete flavor symmetries. We also consider the generation of $U_{l} \simeq V_{\mathrm{CKM}}$.

The paper is organized as follows. In Sec. II, we describe the scenario in details. In Sec. III, we study the possibility to generate the matrix $U_{0}$ using the residual symmetry approach applied to the visible and hidden sectors. We find all possible structures of $U_{0}$. In Sec. IV, we present the realization of the residual symmetry mechanism that generates $U_{0}=U_{\mathrm{BM}}$ and is based on the $S_{4}$ symmetry group. We explore the possibility of generating the CKM mixing and study the predictions for the Pontecorvo-MakiNakagawa-Sakata (PMNS) mixing in Sec. V. Conclusions are given in Sec. VI.

\section{FRAMEWORK}

Let us describe the main elements of the framework.

\section{A. Visible, portal, and hidden sectors}

The visible sector includes three families of fermions accommodated in three 16-plets of $\mathrm{SO}(10):\left(\psi_{1}, \psi_{2}, \psi_{3}\right)$. The fermions get masses mainly via the Yukawa couplings with a 10-plet scalar field $H^{(10)}$. Additional nonrenormalizable interactions will be added to generate the difference of masses of down quarks and charged leptons.

The hidden sector consists of fermions $S_{i}$ and bosons $\phi_{i}$, which are all singlets of $\mathrm{SO}(10)$. In the simplest version, three fermionic singlets are introduced. A connection between the visible and hidden sectors is established via the portal interaction of $S_{i}$ and $\psi_{i}$. For this, the 16-plet of scalar fields, $H^{(16)}$, should be introduced.

Thus, the Yukawa interactions relevant for generation of fermion masses are

$$
\mathcal{L} \supset y_{i j}^{v} \psi_{i} \psi_{j} H^{(10)}+y_{i j}^{p} \psi_{i} S_{j} H^{(16)}+h_{i j} S_{i} S_{j} \phi_{i j},
$$

where $y_{i j}^{v}, y_{i j}^{p}$, and $h_{i j}$ are the Yukawa coupling constants of the visible, portal, and hidden sectors, correspondingly.

\section{B. Double seesaw}

After the scalar fields develop vacuum expectation values (VEVs), the visible sector interactions generate the Dirac mass matrices $m_{D}=y_{i j}^{v}\left\langle H^{(10)}\right\rangle$ at the electroweak (EW) scale. The portal interactions produce the matrix $M_{R S}=y_{i j}^{p}\left\langle H^{(16)}\right\rangle$, which mixes the RH neutrinos with the singlet fermions. ${ }^{2}$ Flavon VEVs $\left\langle\phi_{i j}\right\rangle$ generate the mass

\footnotetext{
${ }^{2} \mathrm{~A}$ linear seesaw contribution $m_{L S}$ (see Ref. [37]) can also be generated, but in this framework, its contribution is negligibly small.
} 
matrix of singlets $M_{S i j}=h_{i j}\left\langle\phi_{i j}\right\rangle$. Consequently, the total mass matrix of neutral leptons in the basis $\left(\nu_{L}, \nu_{L}^{c}, S\right)$ [here, $\left.\nu_{L}^{c} \equiv\left(\nu_{R}\right)^{c}\right]$ becomes

$$
\mathcal{M}=-\frac{1}{2}\left(\begin{array}{ccc}
0 & m_{D} & 0 \\
m_{D}^{T} & 0 & M_{R S} \\
0 & M_{R S}^{T} & M_{S}
\end{array}\right),
$$

which is the mass matrix of the double-seesaw mechanism [40]. It leads to the Majorana masses of the RH neutrinos $\nu_{R}$,

$$
M_{R}=-M_{R S} M_{S}^{-1} M_{R S}^{T},
$$

and the mass matrix of light neutrinos $\nu_{L}$,

$$
m_{\nu}=m_{D}\left(M_{R S}^{-1}\right)^{T} M_{S} M_{R S}^{-1} m_{D}^{T}
$$

The Higgs multiplet $H^{(16)}$ breaks the grand unified theory (GUT) symmetry, so the natural scale of the largest portal mass term is $M_{R S} \sim M_{\mathrm{GUT}}=\mathcal{O}\left(10^{16} \mathrm{GeV}\right)$. The singlets $S$, which are not protected by $\mathrm{SO}(10)$, may have masses of higher scales, say, the string-Planck scale, $M_{S} \sim M_{\mathrm{Pl}} \sim\left(10^{18}-10^{19}\right) \mathrm{GeV}$. In this case, $M_{R}=$ $M_{\mathrm{GUT}}^{2} / M_{\mathrm{Pl}}=\mathcal{O}\left(10^{14} \mathrm{GeV}\right)$, which produces the correct scale of light neutrino masses $m_{\nu}=\mathcal{O}(0.1 \mathrm{eV})$. This coincidence can be considered as another support of the framework.

\section{Intrinsic symmetries}

Both the visible and hidden sector interactions (4) have the built-in $\mathbb{Z}_{2} \times \mathbb{Z}_{2} \times \mathbb{Z}_{2}$ flavor symmetries [41-44]. The symmetries are related to the Majorana character of interaction $\left(\psi^{T} \cdot \psi, S^{T} \cdot S\right)$ and are obvious in the basis in which the mass matrices are diagonalized. In a general basis, the visible interactions are invariant under the transformation

$\psi \rightarrow T \psi, \quad T=U_{\psi} \operatorname{diag}\left[(-1)^{m},(-1)^{n},(-1)^{k}\right] U_{\psi}^{\dagger}$,

where $m, n, k=0,1$, and $U_{\psi}$ is a unitary matrix that diagonalizes $m_{D}$. Different choices of $m, n, k$ generate different $T$ 's, including trivial cases $T= \pm \mathbb{I}$. These $T$ 's form the $\mathbb{Z}_{2} \times \mathbb{Z}_{2} \times \mathbb{Z}_{2}$ group, which can be reduced to $G_{V}=\mathbb{Z}_{2} \times \mathbb{Z}_{2}$ if generators with the overall negative sign are removed. Similarly, in the hidden sector, the mass terms $1 / 2\left(M_{S}\right)_{i j} S_{i} S_{j}$ are invariant under

$S \rightarrow R S, \quad R=U_{S} \operatorname{diag}\left[(-1)^{m},(-1)^{n},(-1)^{k}\right] U_{S}^{\dagger}$,

where $U_{S}$ is a unitary matrix that diagonalizes $M_{S}$. So, the hidden sector has another $\mathbb{Z}_{2} \times \mathbb{Z}_{2}$ symmetry, which is denoted as $G_{H}$ henceforth.

\section{Screening}

The condition

$$
m_{D} \propto M_{R S}^{T}
$$

leads, as follows from Eq. (7), to

$$
m_{\nu} \propto M_{S}
$$

i.g., to the "screening" (cancellation) of the Dirac structures and, consequently, to the same structure of the mass matrices of light neutrinos and the heavy singlets [33]. Consequently, the light neutrinos and the heavy singlets have the same mixing. The renormalization group equation (RGE) effects do not destroy the cancellation [33].

The screening condition (10) can be a consequence of further unification, e.g., embedding of $\psi$ and $S$ into the 27-dimensional representation of the $E_{6}$-multiplet. It can be a remnant of $E_{6}$ symmetry, which is not fully realized. In this case, $S$ cannot be considered as belonging to the hidden sector. Another possibility is a common flavor symmetry acting in the visible and portal sectors. In fact, it is easy to get

$$
m_{D} M_{R S}^{-1 T}=d,
$$

where $d$ is a diagonal matrix. The Klein symmetry $\mathbb{Z}_{2} \times \mathbb{Z}_{2}$ with the same charge assignment for $\phi_{i}$ and $S_{i}$ allows one to achieve this. If the charges of three components $\psi_{1}, \psi_{2}$, and $\psi_{3}$ are different, e.g., $(-,-)(-,+)$, and $(+,-)$ and scalar multiplets $H^{(10)}$ and $H^{(16)}$ have zero charges, the matrices $m_{D}$ and $M_{R S}$ are both diagonal [37]. However, additional symmetry should be introduced to make the ratios of the element in $m_{D}$ and $M_{R S}$ to be equal so that $d=\mathbb{I}$. A kind of permutation symmetry $\psi \leftrightarrow S$ could be used.

\section{E. Basis-fixing symmetry}

$G_{b}=\mathbb{Z}_{2} \times \mathbb{Z}_{2}$ was introduced as the basis-fixing symmetry in all the sectors, thus allowing one to communicate information about mixing from the hidden sector to the visible one. This symmetry leads to the diagonal structure of all Dirac mass matrices, even if several Higgs 10-plets (or other representations) with zero charges are introduced. This means that no CKM mixing is generated, $U_{\mathrm{CKM}}=\mathbb{I}$. Therefore, generation of CKM mixing would require the breaking of the $\mathbb{Z}_{2} \times \mathbb{Z}_{2}$ symmetry. $G_{b}$ can be identified with $G_{V}$. To generate mixing $U_{0}, G_{b}$ should be broken in the hidden sector.

\section{F. Flavons}

The key element of the framework is that scalars of the hidden sector $\phi$ do carry nontrivial $G_{b}$ charges, in contrast to $H^{(10)}$ and $H^{(16)}$. When $\phi_{i}$ get VEVs, $G_{b}$ is spontaneously broken in the hidden sector. This leads to nondiagonal 
matrix $M_{S}$ and, consequently, to the mixing of singlets $S_{i}$. It is this mixing that generates the matrix $U_{0}$. The Klein (Abelian) symmetry is not enough to obtain a special form of $M_{S}$ with a fully determined mixing matrix like TBM. For this, non-Abelian symmetry should be introduced in the hidden sector.

\section{III. $U_{0}$ FROM THE RESIDUAL SYMMETRIES OF VISIBLE AND HIDDEN SECTORS}

In the case of complete screening, the portal interactions do not influence the mixing, and one can immediately discuss the bases of the visible and hidden sectors. Then, the mixing can be understood as a relation between the bases in which generators of $G_{V}$ and $G_{H}$ have diagonal forms. According to Eqs. (8) and (9), these two bases should be connected by $U_{0}=U_{\psi}^{\dagger} U_{S}$. Thus, we start with common basis-fixing symmetry $G_{b}$ in all the sectors and then promote $G_{b}$ to a larger non-Abelian group $G_{f}$ in the hidden sector. Then, the breaking of $G_{f}$ should be arranged in such a way that $G_{b} \in G_{f}$ is broken and another intrinsic unbroken $G_{H}=\left(\mathbb{Z}_{2} \times \mathbb{Z}_{2}\right)^{H}$ symmetry is realized.

One can view this procedure as the residual symmetry approach: $G_{H}$ and $G_{V}$ are embedded into a unified flavor group,

$$
G_{f} \supset G_{H}, G_{V} .
$$

This embedding ensures that information about mixing from the hidden sector is transmitted to the visible sector. Then, $G_{f}$ is broken explicitly down to $G_{V}$ in the visible (low-mass scale) sector, and it is broken down to $G_{H}$ spontaneously in the hidden sector. ${ }^{3}$

This is similar to the usual residual symmetry approach [41-44] when, instead of mass matrices of the charged leptons and neutrinos, we use the mass matrices of $\psi$ and $S$. Here, the residual symmetries operate at different energy scales: the GUT scale and the Planck scale.

An important feature is that in both sectors the residual symmetries are given by the Klein groups:

$$
G_{V}=\left(\mathbb{Z}_{2} \times \mathbb{Z}_{2}\right)^{V}, \quad G_{H}=\left(\mathbb{Z}_{2} \times \mathbb{Z}_{2}\right)^{H} .
$$

The embedding of two Klein groups into a finite group and its consequences for mixing have been explored in Ref. [39]. The only difference is that in Ref. [39] the results of embedding were applied to the relative matrix between interactions with a Higgs 10-plet and Higgs 126plet, while here we deal with the relative rotation between the mass basis generated by the Higgs 10-plet (visible sector that coincides with the matrix of the portal) and the

\footnotetext{
${ }^{3}$ One can consider also spontaneous symmetry breaking in the visible and portal sectors, but this would introduce further complication of the model.
}

mass basis generated by Higgs singlets in the hidden sector. In what follows, we briefly remind the reader of the important points and present the main results.

In a three-dimensional irreducible representation of $G_{f}$, the elements $T \in G_{V}$ and $R \in G_{H}$ in Eqs. (8) and (9) with positive determinants can be written as

$$
\begin{gathered}
T_{1}=\left(\begin{array}{lll}
1 & & \\
& -1 & \\
& & -1
\end{array}\right), \\
T_{2}=\left(\begin{array}{lll}
-1 & & \\
& 1 & \\
& & -1
\end{array}\right), \\
T_{3}=\left(\begin{array}{lll}
-1 & & \\
& -1 & \\
& & 1
\end{array}\right), \\
R_{1}=U_{0}\left(\begin{array}{lll}
1 & & \\
& -1 & \\
& & -1
\end{array}\right) U_{0}^{\dagger}, \\
R_{2}=U_{0}\left(\begin{array}{lll}
-1 & & \\
& 1 & \\
& & -1
\end{array}\right) U_{0}^{\dagger}, \\
R_{3}=U_{0}\left(\begin{array}{lll}
-1 & & \\
& -1 & \\
& & 1
\end{array}\right) U_{0}^{\dagger},
\end{gathered}
$$

where we use the basis in which $T$ is diagonal. By definition, the group $G_{f}$ should contain all these elements as well as their products. Furthermore, since $G_{f}$ is a finite group, any product of $T$ and $R$ should have a finite order,

$W_{i j}^{p} \equiv\left(T_{i} R_{j}\right)^{p}=\left(T_{i} U_{0} R_{j}^{d} U_{0}^{\dagger}\right)^{p}=\mathbb{I}, \quad(i, j=1,2,3)$,

where $p$ is a positive integer. This is the symmetry group condition [39] that determines the $i-j$ element of $U_{0}$,

$$
\left|\left(U_{0}\right)_{i j}\right|^{2}=\cos ^{2} \frac{\pi n}{p}
$$

where $n$ and $p$ are integers. The detailed derivation of Eq. (17) is presented in Appendix A. Using one generator $T_{i} \in G_{V}$ and another one $R_{j} \in G_{H}$ fixes the $i$ - $j$ element of the matrix $U_{0}$. The indices $i, j=1,2,3$ are identified by the positive diagonal elements in Eqs. (14) and (15). For instance, $i=1$ corresponds to generators with +1 in the 1-1 position, etc. 
Each pair of $(i, j)$ has its own power $p_{i j}$, which should be larger than $p_{i j}$. Furthermore, for fixed $p_{i j}$, several $n_{i j}$ can exist. Taking three different symmetry group relations given by one $T$ and three $R$ (or vice versa), we can fix three elements of the row (column) of the mixing matrix, and they should satisfy the unitarity condition. Using Eq. (17), we can write the unitarity condition, in general, as

$$
\cos ^{2} \alpha+\cos ^{2} \beta+\cos ^{2} \gamma=1
$$

where $\alpha, \beta$, and $\gamma$ are rational numbers of $\pi$.

As we will see, the unitarity condition in the form (18) already strongly restricts the number of possibilities even before further applications of the group theory constraints. Without loss of generality, we assume that $\cos \alpha \leq \cos \beta \leq$ $\cos \gamma$ and $0 \leq \alpha, \beta, \gamma \leq 90^{\circ}$. Under these assumptions, we only need to consider two cases: $\cos \alpha=0$ and $\cos \alpha \neq 0$.

If $\cos \alpha$ is zero $(\alpha=\pi / 2)$, Eq. (18) reduces to $\cos ^{2} \beta+\cos ^{2} \gamma=1$, from which one immediately obtains $\gamma=\pi / 2-\beta$. Since $\beta=\pi q / p$, we get an infinite number of solutions for the angles:

$$
(\alpha, \beta, \gamma)=\pi\left(\frac{1}{2}, \frac{q}{p}, \frac{1}{2}-\frac{q}{p}\right) .
$$

For $\cos \alpha \neq 0$ (all cosines are nonzero), a numerical search for all rationals $q / p$ with $p \leq 100$ gives only two solutions:

$$
(\alpha, \beta, \gamma)=\pi\left(\frac{1}{3}, \frac{1}{3}, \frac{1}{4}\right)
$$

and

$$
(\alpha, \beta, \gamma)=\pi\left(\frac{2}{5}, \frac{1}{3}, \frac{1}{5}\right)
$$

The elements of $\left|U_{0}\right|$ that correspond to Eqs. (19)-(21) are

$$
\begin{aligned}
& v_{1} \equiv\left(0, \cos \frac{q}{p} \pi, \sin \frac{q}{p} \pi\right), \quad v_{2} \equiv\left(\frac{1}{\sqrt{2}}, \frac{1}{2}, \frac{1}{2}\right), \\
& v_{3} \equiv\left(\frac{\sqrt{5}+1}{4}, \frac{1}{2}, \frac{\sqrt{5}-1}{4}\right) .
\end{aligned}
$$

The last solution in Eq. (22) can be expressed in terms of the golden ratio,

$$
\varphi \equiv \frac{1}{2}(1+\sqrt{5}) \approx 1.618
$$

$v_{3}=\frac{1}{2}\left(\varphi, 1, \varphi^{-1}\right)^{T}$. The first solution in Eq. (22) has two interesting possibilities:

$$
v_{1 a}=(1,0,0), \quad v_{1 b}=\left(\frac{1}{\sqrt{2}}, \frac{1}{\sqrt{2}}, 0\right)
$$

Now, using the vectors in Eq. (22) as building blocks, we can construct complete mixing matrices. There is the freedom to take $v_{i}$ as rows or columns of the matrix and also to permute elements within $v_{i}$. Not all combinations are allowed by unitarity. If the unitarity is satisfied for the column, one should arrange the elements in each column so that it is satisfied for rows as well. Clearly, using the same column three times with permuted elements will automatically satisfy the unitarity condition for whole the matrix.

Let us consider first that at least one of the columns of $\left|U_{0}\right|$ is in the form of $v_{1}$ in Eq. (22), which means that $U_{0}$ has at least one zero entry. In general, one can prove ${ }^{4}$ that the number of zero entries in a $3 \times 3$ unitary matrix can only be 1 or 4 or 6 . The matrix with four zeros constructed of $v_{1}$ is

$$
\left|U_{0}\right|=\left(\begin{array}{ccc}
1 & 0 & 0 \\
0 & \cos \frac{q}{p} \pi & \sin \frac{q}{p} \pi \\
0 & \sin \frac{q}{p} \pi & \cos \frac{q}{p} \pi
\end{array}\right) .
$$

The case of six zeros corresponds to $\left|U_{0}\right|=I$.

For the case of a single zero, we take $v_{1}$, while the other columns cannot contain zero entries, and therefore they have to be of the form $v_{2}$ or $v_{3}$. The conclusion about the columns of $\left|U_{0}\right|$ also holds for the rows, which implies that the rows consist of one $v_{1}^{T}$ and two $v_{2}^{T}$ or $v_{3}^{T}$. As a result, the two nonzero elements in $v_{1}$ have to be $1 / 2,1 / \sqrt{2}, \varphi / 2$, or $\varphi^{-1} / 2$. The squared sum of the two nonzero elements should be 1 , leaving only one option: $(0,1 / \sqrt{2}, 1 / \sqrt{2})$. Therefore, in the case of 1 zero, we have

$$
\left|U_{0}\right|=\left(\begin{array}{ccc}
1 / \sqrt{2} & 1 / \sqrt{2} & 0 \\
1 / 2 & 1 / 2 & 1 / \sqrt{2} \\
1 / 2 & 1 / 2 & 1 / \sqrt{2}
\end{array}\right)
$$

which coincides with the BM mixing matrix [7,45-49]. There are no other possibilities with $v_{1}$.

Next, let us consider $\left|U_{0}\right|$ constructed from $v_{2}$ or $v_{3}$ or $v_{2}$ and $v_{3}$ together. There is only one solution in each case:

\footnotetext{
${ }^{4}$ The proof is straightforward enumeration. The number of zeros cannot be larger than six because it implies that at most two elements of $U_{0}$ can be nonzero, which is impossible for a unitary matrix. If there are five zeros, i.e., four elements are nonzero, then the orthogonality of rows/columns requires one of the four elements to be zero. Likewise, one can check that $U_{0}$ with two or three zeros has the same problem.
} 


$$
\begin{array}{rlrl}
\left|U_{0}\right| & =\frac{1}{2}\left(\begin{array}{ccc}
\sqrt{2} & 1 & 1 \\
1 & \sqrt{2} & 1 \\
1 & 1 & \sqrt{2}
\end{array}\right), & \frac{1}{2}\left(\begin{array}{ccc}
\varphi & 1 & \varphi^{-1} \\
1 & \varphi^{-1} & \varphi \\
\varphi^{-1} & \varphi & 1
\end{array}\right), \\
& \frac{1}{2}\left(\begin{array}{ccc}
\varphi^{-1} & 1 & \varphi \\
1 & \sqrt{2} & 1 \\
\varphi & 1 & \varphi^{-1}
\end{array}\right) .
\end{array}
$$

The symmetry group condition gives the moduli of elements, $\left|U_{0}\right|$. To reconstruct $U_{0}$ completely, one needs to find the phases of elements that ensure the orthogonality of the rows and columns in $U_{0}$. Without loss of generality, we assume that $u_{11}, u_{12}, u_{13}, u_{23}$, and $u_{33}$ are real. Then, the orthogonality of the columns gives

$$
\begin{aligned}
& u_{11} u_{13}+\left|u_{21}\right| e^{i \phi_{21}} u_{23}+\left|u_{31}\right| e^{i \phi_{31}} u_{33}=0, \\
& u_{12} u_{13}+\left|u_{22}\right| e^{i \phi_{22}} u_{23}+\left|u_{32}\right| e^{i \phi_{32}} u_{33}=0,
\end{aligned}
$$

where $\phi_{i j} \equiv \arg \left(u_{i j}\right)$. Using the graphic representation of the equalities (28) and (29) (i.e., the unitarity triangles), we obtain

$$
\begin{aligned}
& \cos \phi_{2 j}=\frac{\left|u_{3 j}\right|^{2} u_{33}^{2}-u_{1 j}^{2} u_{13}^{2}-\left|u_{2 j}\right|^{2} u_{23}^{2}}{2 u_{1 j} u_{13}\left|u_{2 j}\right| u_{23}}, \\
& \cos \phi_{3 j}=\frac{\left|u_{2 j}\right|^{2} u_{23}^{2}-u_{1 j}^{2} u_{13}^{2}-\left|u_{3 j}\right|^{2} u_{33}^{2}}{2 u_{1 j} u_{13}\left|u_{3 j}\right| u_{33}}, \quad(j=1,2) .
\end{aligned}
$$

Consequently, the phases for the three matrices in Eq. (27) equal

$$
\begin{aligned}
\arg \left(U_{0}\right) & =\left(\begin{array}{ccc}
0 & 0 & 0 \\
\arccos \frac{-1}{2 \sqrt{2}} & -\arccos \frac{-3}{4} & 0 \\
-\arccos \frac{-3}{4} & \arccos \frac{-1}{2 \sqrt{2}} & 0
\end{array}\right), \\
& \left(\begin{array}{ccc}
0 & 0 & 0 \\
\pi & -\pi & 0 \\
0 & \pi & 0
\end{array}\right), \\
& \left(\begin{array}{ccc}
0 & 0 & 0 \\
2 \pi / 3 & -\arccos (\sqrt{2}(\sqrt{5}-3))^{-1} & 0 \\
-2 \pi / 3 & 2 \pi / 3 & 0
\end{array}\right) .
\end{aligned}
$$

The matrices in Eqs. (25) and (26) contain zero mixing angles, and therefore the phases can be removed by rephasing. Orthogonality in Eqs. (25) and (26) can be achieved by adding minus signs, e.g., to 2-1, 3-1, and 2-3 elements in Eq. (26) and to the 3-2 element in Eq. (25). The mixing matrices including the phases are summarized in Table I.

TABLE I. All rational mixing matrices with $p \leq 100$ and the corresponding finite groups.

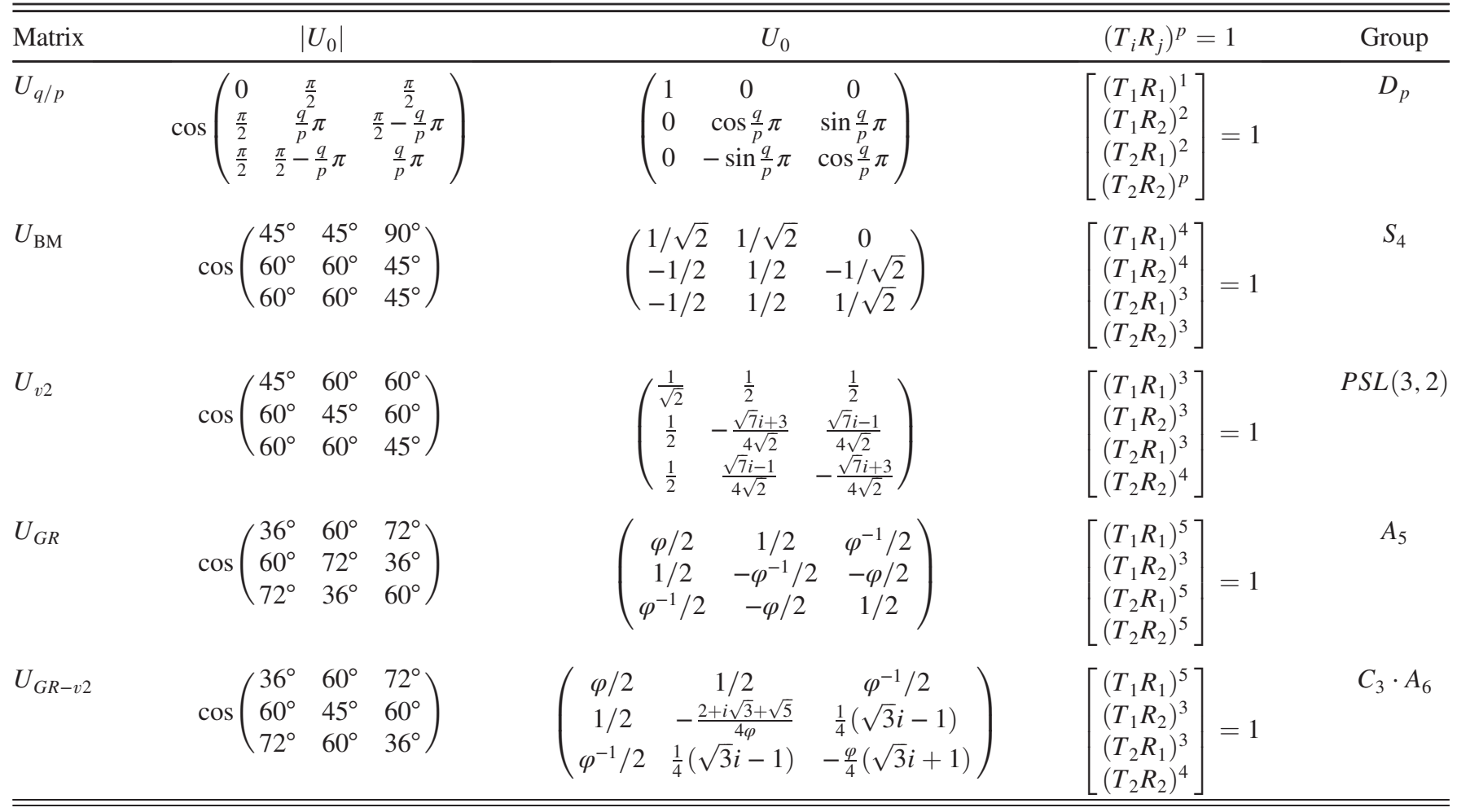


Notice that until now we used only a general form of matrix elements as cosines of rational numbers of $\pi$ (17) and the unitarity. The symmetry group condition is necessary but not sufficient for embedding into the finite group. Still, one should check that embedding is possible from the group theory point of view. Using $U_{0}$ given in the Table I, we can find the corresponding generators $R_{i}$ according to Eq. (15) and check the group presentations. Then, using the GAP program [50], we identify all the corresponding finite groups (see the last column of Table I). Notice that the block-diagonal matrices $U_{q / p}$ are generated by a dihedral group $D_{p}$, of which the order $p$ is determined by the denominator of the rational angle. The matrices $U_{\mathrm{BM}}, U_{v 2}$, and $U_{G R}$ are generated by the groups $S_{4}, \operatorname{PSL}(3,2)$, and $A_{5}$, of orders 24,168 , and 60 , correspondingly. It has been well known (see, e.g., Refs. $[48,49]$ ) that the $S_{4}$ symmetry can be used to obtain $U_{\mathrm{BM}}$. The last and most complicated matrix $U_{G R-v 2}$ can be obtained in a 1080-order group, which is a nonsplit extension of $A_{6}$ by $C_{3}$, denoted as $C_{3}$. $A_{6}$ in the GAP classification system.

Reconstructing finite groups from the residual symmetries has been studied Ref. [51] using theorems on sums of roots of unity, which is technically similar to the trace approach formulated in Eq. (A4). Some of the finite groups presented in the Table I (e.g., $S_{4}$ and $A_{5}$ ) are the same as those found in Ref. [51]. However, one should note that Ref. [51] sets a finite order of $T^{\dagger} R T R$, while in Eq. (16), we use the order of $T R$. Consequently, we obtain some additional groups such as $\operatorname{PSL}(3,2)$ and $C_{3} \cdot A_{6}$.

In summary, the BM mixing matrix can be obtained for $U_{0}$ in our approach. Also, the matrices $U_{v 2}$ (constructed with columns $\left.v_{2}, v_{2}, v_{2}\right)$ and $U_{G R}\left(v_{3}, v_{3}, v_{3}\right)$ can be of the phenomenological interest once certain corrections are taken into account. The matrix $U_{q / p}$ can be considered for the 2-3 mixing if the 1-2 mixing is generated, e.g., from the portal interactions. Notice that the TBM mixing cannot be obtained in this framework. This is because we require that the residual symmetries should be $\mathbb{Z}_{2} \times \mathbb{Z}_{2}$ while TBM actually needs a $\mathbb{Z}_{3}$ residual symmetry. Without the requirement of $\mathbb{Z}_{2} \times \mathbb{Z}_{2}$, TBM may be obtained in SO (10) frameworks—see, e.g., Ref. [52].

\section{BIMAXIMAL MIXING FROM THE HIDDEN SECTOR}

For definiteness, we consider generating the BM mixing from the $S_{4}$ embedding of the residual symmetries. Details of the group $S_{4}$, which has five irreducible representations $\mathbf{1}, \mathbf{1}^{\prime}, \mathbf{2}, \mathbf{3}$, and $\mathbf{3}^{\prime}$, are given in Appendix B. All the fermions are assigned to the three-dimensional representation $\mathbf{3}$. The SO(10) Higgs multiplets $H^{(10)}$ and $H^{(16)}$ are flavor singlets. In contrast, the Higgs fields in the hidden sector have
TABLE II. Field content of the model and symmetry assignments.

\begin{tabular}{lccccccc}
\hline \hline & $\psi$ & $S$ & $H^{(10)}$ & $H^{(16)}$ & $\eta$ & $\xi$ & $\phi$ \\
\hline Type & Fermion & Fermion & Scalar & Scalar & Scalar & Scalar & Scalar \\
SO(10) & $\mathbf{1 6}$ & $\mathbf{1}$ & $\mathbf{1 0}$ & $\mathbf{1 6}$ & $\mathbf{1}$ & $\mathbf{1}$ & $\mathbf{1}$ \\
$S_{4}$ & $\mathbf{3}$ & $\mathbf{3}$ & $\mathbf{1}$ & $\mathbf{1}$ & $\mathbf{1}$ & $\mathbf{2}$ & $\mathbf{3}^{\prime}$ \\
\hline \hline
\end{tabular}

nontrivial $S_{4}$ assignments, and so the flavor symmetry is broken in this sector spontaneously. The symmetry assignments for the fields are given in Table II.

We assume that in the visible and portal sectors the $S_{4}$ symmetry is broken explicitly down to the residual symmetry $\left(\mathbb{Z}_{2} \times \mathbb{Z}_{2}\right)^{V}$. The $\left(\mathbb{Z}_{2} \times \mathbb{Z}_{2}\right)^{V}$ charges of $\psi$ and $S$ are

$$
\begin{array}{clll}
\text { Fields: } & \psi_{1}, S_{1} & \psi_{2}, S_{2} & \psi_{3}, S_{3} \\
\left(\mathbb{Z}_{2} \times \mathbb{Z}_{2}\right)^{V}: & (+,-) & (-,+) & (-,-)
\end{array} .
$$

The visible and portal sectors are invariant under the transformation

$$
\psi \rightarrow T_{i} \psi, \quad S \rightarrow T_{i} S, \quad(i=1,2),
$$

where $T_{i}$ are defined in Eq. (14), and the transformations in Eq. (31) belong to a subgroup of $S_{4}$.

Because of the $\left(\mathbb{Z}_{2} \times \mathbb{Z}_{2}\right)^{V}$ symmetry (31), the Yukawa interactions in the visible and portal sectors are flavordiagonal, so the Lagrangian (4) reduces to

$$
\mathcal{L}_{\psi}=\sum_{i=1}^{3}\left[y_{i}^{v} \psi_{i} \psi_{i} H^{(10)}+y_{i}^{p} \psi_{i} S_{i} H^{(16)}\right]
$$

In the hidden sector, the Yukawa interactions are

$$
\mathcal{L}_{\text {hidden }}=y_{i j k}^{\phi} S_{i} S_{j} \phi_{k}+y_{i j k}^{\xi} S_{i} S_{j} \xi_{k}+y^{\eta} S_{i} S_{i} \eta
$$

where the Yukawa couplings $y_{i j k}^{\phi}$ and $y_{i j k}^{\xi}$ are determined by the $S_{4}$ symmetry. According to the Clebsch-Gordan coefficients of $S_{4}$ (see Appendix B), the products of these Yukawa couplings with the flavon fields (which eventually determine the mass matrix of $S$ ) can be expressed in the following matrix forms in the basis $\left(S_{1}, S_{2}, S_{3}\right)^{T}$ :

$$
\sum_{k} y_{i j k}^{\phi} \phi_{k}=y^{\phi}\left(\begin{array}{ccc}
0 & \phi_{2}-\phi_{3} & -\phi_{2}-\phi_{3} \\
\phi_{2}-\phi_{3} & \sqrt{2} \phi_{1} & 0 \\
-\phi_{2}-\phi_{3} & 0 & -\sqrt{2} \phi_{1}
\end{array}\right) \text {, }
$$

and 


$$
\sum_{k} y_{i j k}^{\xi} \xi_{k}=y^{\xi}\left(\begin{array}{ccc}
-e^{\frac{i \pi}{6}} \xi_{1}-\frac{\xi_{2}}{\sqrt{3}} & 0 & 0 \\
0 & \frac{1}{6}\left(3 e^{\frac{i \pi}{6}} \xi_{1}+\sqrt{3} \xi_{2}\right) & \frac{1}{2}\left(e^{\frac{i \pi}{6}} \xi_{1}-\sqrt{3} \xi_{2}\right) \\
0 & \frac{1}{2}\left(e^{\frac{i \pi}{6}} \xi_{1}-\sqrt{3} \xi_{2}\right) & \frac{1}{6}\left(3 e^{\frac{i \pi}{6}} \xi_{1}+\sqrt{3} \xi_{2}\right)
\end{array}\right)
$$

To obtain nontrivial flavor structures, $S_{4}$ should be broken down to $\left(\mathbb{Z}_{2} \times \mathbb{Z}_{2}\right)^{H}$, which differs from $\left(\mathbb{Z}_{2} \times \mathbb{Z}_{2}\right)^{V}$. $\left(\mathbb{Z}_{2} \times \mathbb{Z}_{2}\right)^{H}$ is represented by the matrices $R(15)$ in $\mathbf{3}$ of $S_{4}$ and by $R^{(\mathbf{1})}, R^{\left(\mathbf{1}^{\prime}\right)}, R^{\left(\mathbf{2}^{\prime}\right)}$, and $R^{\left(\mathbf{3}^{\prime}\right)}$ in the representations $\mathbf{1}, \mathbf{1}^{\prime}, \mathbf{2}$, and $\mathbf{3}^{\prime},\left(\mathbb{Z}_{2} \times \mathbb{Z}_{2}\right)^{H}$ (see the Appendix). Since the flavons $\phi$ and $\xi$ are assigned to $\mathbf{3}^{\prime}$ and $\mathbf{2}$ and break $S_{4}$ down to $\left(\mathbb{Z}_{2} \times \mathbb{Z}_{2}\right)^{H}$, their VEVs should be invariant under $\left(\mathbb{Z}_{2} \times \mathbb{Z}_{2}\right)^{H}$, i.e.,

$$
R^{\left(3^{\prime}\right)}\langle\phi\rangle=\langle\phi\rangle, \quad R^{(2)}\langle\xi\rangle=\langle\xi\rangle .
$$

This gives

$$
\langle\phi\rangle \propto(0,0,1)^{T}, \quad\langle\xi\rangle \propto(0,1)^{T},
$$

where we used explicit forms of $R^{\left(3^{\prime}\right)}$ and $R^{(2)}$ from Eqs. (B4) and (B5). The potentials that produce the vacuum alignment (37) can be easily constructed [48]. Finally from Eqs. (34), (35), and (37), we obtain the explicit form of $M_{S}$, and consequently $m_{\nu}$,

$$
m_{\nu} \propto M_{S}=\left(\begin{array}{ccc}
a-2 c & b & b \\
b & a+c & -3 c \\
b & -3 c & a+c
\end{array}\right),
$$

where

$$
a=y^{\eta}\langle\eta\rangle, \quad b=y^{\phi}\langle\phi\rangle, \quad c=\frac{y^{\xi}}{2}\langle\xi\rangle .
$$

The mass matrix in Eq. (38) is diagonalized by $U_{0}=$ $U_{\mathrm{BM}}$ with the eigenvalues

$U_{0}^{T} m_{\nu} U_{0}=\left(\begin{array}{ccc}a-\sqrt{2} b-2 c & 0 & 0 \\ 0 & a+\sqrt{2} b-2 c & 0 \\ 0 & 0 & a+4 c\end{array}\right)$.

The three parameters $a, b$, and $c$ are sufficient to fit three light neutrino masses.

\section{CKM MIXING AND PMNS MIXINGS}

Generation of $U_{0}$ and screening in our framework require that the CKM-type mixing originates from the down components of the EW doublets. Therefore, to reproduce the relation (1), the mixing of the charged leptons should be approximately equal to the down-quark mixing:
$U_{l} \approx V_{\mathrm{CKM}} .{ }^{5}$ This approximate equality of mixings should be reconciled with the difference of masses of down quarks and charged leptons in the second and the first generations. In fact, according to the two-loop RGE running in the standard model [53], we have at the GUT scale

$$
m_{\mu} \approx 3 m_{s} \gg m_{e}, m_{d}
$$

This problem was extensively discussed before in connection to the quark-lepton complementarity [16]. Actually, in the case of strong mass hierarchy, the difference of mixings related to the difference of masses is not large and may be even needed to better fit of the data.

In general, there are two approaches to keep the relation $U_{l} \approx U_{d}$ for different masses. One is to decouple completely the generation of masses from mixing so that the mixing comes from certain relations between the elements of the mass matrix, whereas the masses are determined by absolute values of the elements. This decoupling is difficult to obtain for small CKM mixing; simple discrete symmetries usually lead to large nonzero angles. ${ }^{6}$ Hence, this approach would require substantial complications of the model. Another possibility, which we will implement here, is that one of the Higgs multiplets dominates in the generation of charged fermion masses so that $M_{d}$ and $M_{l}$ have roughly the same form, and thus approximately equal mixing. One can also add a mass matrix proportional to the unit matrix; this does not change the mixing but affects the mass ratio.

With one 10-plet, which conserves the basis-fixing symmetry, we obtain at the GUT scale the diagonal mass matrices

$$
M_{d}^{(10)}=M_{l}^{(10)}=\frac{v_{d}}{v_{u}} M_{u}^{(10)}=\left(\begin{array}{ccc}
k_{1} & 0 & 0 \\
0 & k_{2} & 0 \\
0 & 0 & k_{3}
\end{array}\right),
$$

where $v_{d}$ and $v_{u}$ are the VEVs of the 10-plet that generate masses of the upper and bottom components of the EW

\footnotetext{
${ }^{5}$ We take the following convention in the definitions of $U_{u}, \quad U_{d}, \quad U_{e}$, and $U_{0}: M_{u}=U_{u} \operatorname{diag}\left(m_{u}, m_{c}, m_{t}\right) U_{u}^{T}, M_{d}=$ $U_{d} \operatorname{diag}\left(m_{d}, m_{s}, m_{b}\right) U_{d}^{T}, \quad M_{e}=U_{e} \operatorname{diag}\left(m_{e}, m_{\mu}, m_{\tau}\right) U_{e}^{T}, \quad$ and $m_{\nu}=U_{0}^{*} \operatorname{diag}\left(m_{1}, m_{2}, m_{3}\right) U_{0}^{\dagger}$. In this convention, the CKM and PMNS matrices should be $U_{\mathrm{CKM}}=U_{u}^{\dagger} U_{d}$ and $U_{\mathrm{PMNS}}=$ $U_{l}^{T} U_{0}$.

${ }^{6}$ There are, however, some finite groups that contain small angles—see, e.g., Refs. [54-56].
} 
doublets, correspondingly. The difference of masses of up and down components is due to the difference of VEVs: $v_{d} / v_{u} \approx m_{b} / m_{t}$. Since $k_{i}$ 's are proportional to the quark masses, for $k_{3} \sim m_{b} \sim m_{\tau} \sim 1 \mathrm{GeV}$, we obtain the other masses $k_{2} \approx 3 \mathrm{MeV}$ and $k_{1} \approx 10^{-2} \mathrm{MeV}$, which are much smaller than $m_{s}$ and $m_{d}$, respectively. So, additional sources of mass and mixing are needed. The simplest possibility is that the total matrices of the down-type quarks and charged leptons consist of

$$
M_{d}=M_{d}^{(10)}+M_{x}, \quad M_{l}=M_{d}^{(10)}+a M_{x},
$$

where $|a| \approx 3$ is needed to reproduce (41) and maximal values of elements in $M_{x}$ should be $M_{x}^{\max } \sim m_{s}$ so as not to destroy the $b-\tau$ unification. The matrix $M_{x}$ is nondiagonal, thus breaking the basis-fixing symmetry or $G_{V}$ and producing the CKM mixing.

Correct masses and mixing can be obtained, provided that $M_{x}$ has the structure

$$
M_{x} \approx\left(\begin{array}{ccc}
d_{1} & f & f^{\prime} \\
f & d_{2} & d^{\prime} \\
f^{\prime} & d^{\prime} & d_{3}
\end{array}\right),
$$

with

$$
f^{\prime} \approx f, \quad d^{\prime} \sim d_{2} \sim d_{3}, \quad d_{1} \ll d_{2}, d_{3},
$$

and

$$
f \approx d_{2} \sin \theta_{C}
$$

That is, the mass matrix $M_{x}$ (44) has the dominant 2-3 block and the Cabibbo-suppressed 12 and 13 elements. It is similar to the TBM or BM mass matrices. Therefore, it is also similar to the structure of $M_{S}$ and, consequently, to $m_{\nu}$, in the case of normal mass hierarchy. It is interesting to speculate that common Planck scale physics is responsible for the structure of $M_{S}$ and $M_{x}$.

Numerically, we need to have

$$
d_{i} \sim 0.1 v_{\mathrm{EW}} \frac{M_{\mathrm{GUT}}}{M_{\mathrm{Pl}}} \sim(30-100) \mathrm{MeV},
$$

comparable to the masses of second generation, i.e., the muon and $s$ quark.

The total mass matrices of the down quarks and charged leptons (43) for $a=-3$ become

$$
\begin{aligned}
& M_{d} \approx\left(\begin{array}{ccc}
d_{1}+k_{1} & f & f^{\prime} \\
f & d_{2}+k_{2} & d^{\prime} \\
f^{\prime} & d^{\prime} & d_{3}+k_{3}
\end{array}\right), \\
& M_{l} \approx\left(\begin{array}{ccc}
-3 d_{1}+k_{1} & -3 f & -3 f^{\prime} \\
-3 f & -3 d_{2}+k_{2} & -3 d^{\prime} \\
-3 f^{\prime} & -3 d^{\prime} & -3 d_{3}+k_{3}
\end{array}\right),
\end{aligned}
$$

where $d_{1} \gg k_{1}$ and $d_{2} \gg k_{2}$. So, for the second and the first generations, the contributions from $M_{x}$ dominate. This leads to i) a mass of the muon about three times larger than the mass of the $s$ quark and ii) approximately the same 1-2 mixing of leptons and quarks. For the third generation, the contribution from the 10-plet dominates, $k_{3} \approx h_{3} \gg d_{3}$, thus ensuring the approximate $b-\tau$ unification. In general, $m_{\tau}=m_{b}+\mathcal{O}\left(4 m_{\mu}\right)$.

From Eq. (48), we obtain for the 2-3 quark mixing

$$
V_{c b} \approx \frac{d^{\prime}}{k_{3}} \approx \frac{d^{\prime}}{m_{b}} \approx \frac{m_{s}}{m_{b}}
$$

and the 1-3 quark mixing

$$
V_{u b} \approx \frac{f^{\prime}}{k_{3}} \sim \frac{f}{m_{b}} \sim \frac{m_{s}}{m_{b}} \sin \theta_{C},
$$

in agreement with observations. According to Eqs. (49) and (50), $V_{u b} \sim V_{c b} V_{u s}$.

The lepton mixing parameters are about three times larger:

$$
U_{\mu 3} \approx-\frac{3 d^{\prime}}{m_{\tau}}, \quad U_{e 3} \approx-\frac{3 f^{\prime}}{m_{\tau}}
$$

This corresponds to the angles $\theta_{23}^{l} \sim(4-5)^{\circ}$ and $\theta_{13}^{l} \sim 1^{\circ}$, which give a sizable deviation from maximal 2-3 mixing and observable corrections to the 1-3 mixing.

Decoupling of the third state produces small corrections to the 1-2 submatrix of $M_{d}$ : the correction to the 1-1 element $f^{\prime 2} / m_{b} \sim 0.05 \mathrm{MeV}$, and the relative corrections to other elements are of the order $d^{\prime} / m_{b} \sim m_{s} / m_{b} \sim 3 \%$ and can be neglected. The corresponding relative corrections in the lepton sector are three times larger; the correction to the 1-1 element, which is an order of magnitude larger, $9 f^{\prime 2} / m_{b} \sim 0.45 \mathrm{MeV}$, can be important for the mass of electron. In the 1-2 sector, we can reproduce the GattoSartori-Tonin relation: $\sin \theta_{C} \sim \sqrt{m_{d} / m_{s}}$.

Let us make a few comments on possible origins of $M_{x}$. The straightforward way is to introduce a 126-plet that produces $a=-3$ in Eq. (43). This 126-plet should not contribute substantially to the masses of neutrinos, not to destroy the inverse seesaw with screening. For this, the VEVs of the SU(2) singlet and triplet in the 126-plet should be zero or small. The mass of the 126-plet can be at the Planck scale to avoid the problem of perturbativity of the theory (see, e.g., the review [57]).

Another possibility [58] is to use the composite 126-plet constructed from the product of two 16-plets. The coupling with fermions is given by nonrenormalizable operators suppressed by the Planck scale $M_{\mathrm{Pl}}$ :

$$
\mathcal{L} \supset \frac{1}{M_{\mathrm{Pl}}} \psi \psi H^{(16)} H^{\prime(16)} .
$$


Here, $H^{\prime(16)}$ is the new 16-plet of scalars with zero VEV of the $\mathrm{SU}(2)$ triplet and singlet components. A similar operator with $H^{(16)} H^{(16)}$ can be forbidden by additional symmetry with respect to transformations $H^{(16)} \rightarrow i H^{(16)}$, $H^{\prime(16)} \rightarrow-i H^{\prime(16)}, S \rightarrow-i S$, and $(\eta, \xi, \phi) \rightarrow-(\eta, \xi, \phi)$. Then, one should assume that, due to some Planck scale physics, the down Higgs doublet in the composite 126-plet acquires the VEV

$$
\left\langle\left[H^{(16)} H^{(16)^{\prime}}\right]^{(126)}\right\rangle_{d}=v_{d} M_{\mathrm{GUT}} .
$$

For $v_{d} \sim 0.1 v_{\mathrm{EW}}$, this reproduces Eq. (47).

Notice that, instead of the nonrenormalizable interaction (52), we can introduce

$$
\mathcal{L} \supset \frac{1}{M_{\mathrm{Pl}}} \psi \psi H^{(10)} H^{(45)},
$$

where $H^{(45)}$ is the 45-plet responsible for the $\mathrm{SO}(10)$ symmetry breaking [58]. The product $H^{(10)} H^{(45)}$ contains antisymmetric 120-plet and therefore can remove the degeneracy of charge lepton and d-quark masses. However, the matrix (44) with diagonal elements cannot be reproduced.

In what follows, for simplicity, we will consider mixing of the first two generations only. Inclusion of corrections from the 1-3 or 2-3 mixing changes the following results very little. The mass matrices (48) can be diagonalized by

$U_{d}=\left(\begin{array}{ccc}c & \tilde{s} & 0 \\ -\tilde{s}^{*} & c & 0 \\ 0 & 0 & 1\end{array}\right) P_{d}, \quad U_{l}=\left(\begin{array}{ccc}c_{l} & \tilde{s}_{l} & 0 \\ -\tilde{s}_{l}^{*} & c_{l} & 0 \\ 0 & 0 & 1\end{array}\right) P_{l}$,

where

$$
\begin{aligned}
& c \equiv \cos \theta_{C}, \quad \tilde{s} \equiv \sin \theta_{C} e^{i \phi_{C}}, \\
& c_{l} \equiv \cos \theta_{l}, \quad \tilde{s}_{l}=\sin \theta_{l} e^{i \phi_{l}}
\end{aligned}
$$

and $P_{d}$ and $P_{l}$ are diagonal matrices containing complex phases. Although all elements of the mass matrices in Eq. (48) are complex, for simplicity, we assume that only $f$ is complex. Then, six real parameters $d_{1,2,3}$ and $k_{1,2,3}$ allow us to accommodate the six masses $\left(m_{d}, m_{s}, m_{b}, m_{e}\right.$, $\left.m_{\mu}, m_{\tau}\right)$, while the complex $f$ generates the Cabibbo mixing, $\sin \theta_{C}$, with a complex phase $\phi_{C}$ and analogous mixing $\sin \theta_{l}$ and phase $\phi_{l}$ in the lepton sector.

The phase $\phi_{C}$ has no physical meaning for the $2 \times 2$ form of $U_{d}$. In contrast, as we will see, $\phi_{l}$ is directly related to the $C P$ phase in the PMNS matrix. Introduction of small 1-3 and 2-3 mixing will make $\phi_{C}$ be the origin of $C P$ violation in the CKM mixing. But this will have little effect on the PMNS mixing.
Using the hierarchy $m_{d} \ll m_{s}$ and $m_{e} \ll m_{\mu}$ as well as the smallness of $\sin \theta_{C} \ll 1$, we obtain the approximate relations (for more details, see Appendix C)

$$
\begin{gathered}
\frac{\phi_{l}}{\phi_{C}}=1+\mathcal{O}\left(\frac{m_{d}}{m_{s}}\right), \\
\frac{\sin 2 \theta_{l}}{\sin 2 \theta_{C}} \approx \frac{3\left(m_{s}+m_{d} \cos \phi_{1}\right)}{m_{\mu}+m_{e} \cos \phi_{2}} \approx 1,
\end{gathered}
$$

where

$$
\begin{aligned}
& \phi_{1} \equiv \pi-\arcsin \left(\frac{s^{2} m_{s}}{c^{2} m_{d}} \sin 2 \phi_{l}\right)-2 \phi_{l}, \\
& \phi_{2} \equiv \arcsin \left(\frac{s_{l}^{2} m_{\mu}}{c_{l}^{2} m_{e}} \sin 2 \phi_{l}\right)-2 \phi_{l} .
\end{aligned}
$$

Equation (58) shows the complicated dependence of the phases, on known quantities (fermion masses and the Cabibbo angle) and on $\phi_{l}$, which in turn is related to the leptonic $C P$ phase. Values of $\phi_{1}$ and $\phi_{2}$ for two special values of $\phi_{l}$ can be obtained from Eq. (58),

$\left(\phi_{1}, \phi_{2}\right) \approx \begin{cases}\left(0^{\circ}, 180^{\circ}\right) & \text { for } \phi_{l}= \pm 90^{\circ} \\ \left(180^{\circ}, 0^{\circ}\right) & \text { for } \phi_{l}=0^{\circ} \text { or } 180^{\circ}\end{cases}$

For other values of $\phi_{l}$, results of the numerical study will be presented later.

Equations (56) and (57) show that the charged leptons do have approximately the same mixing as the down-type quarks, $\theta_{l} \approx \theta_{C}$ and $\phi_{l} \approx \phi_{C}$. Recall that the factor 3 in Eq. (57) originates from the effective 126-plets. Taking the $1 \sigma$ range values of $m_{e}, m_{\mu}, m_{u}$, and $m_{s}$ from Ref. [53] and using Eq. (57), we can evaluate the ratio of the angles for the two choices of phases [cf. Eq. (59)]:

$$
\frac{\theta_{l}}{\theta_{C}}= \begin{cases}0.871-1.22 & \text { (i) } \\ 0.999-1.35 & \text { (ii) }\end{cases}
$$

For other values of the phases, one would get intermediate results between those in the cases $\mathrm{i}$ and ii.

According to Eq. (55), the PMNS matrix should be

$$
U_{\mathrm{PMNS}}=\left(\begin{array}{ccc}
c_{l} & -\tilde{s}_{l}^{*} & 0 \\
\tilde{s}_{l} & c_{l} & 0 \\
0 & 0 & 1
\end{array}\right) U_{\mathrm{BM}}
$$

or explicitly 

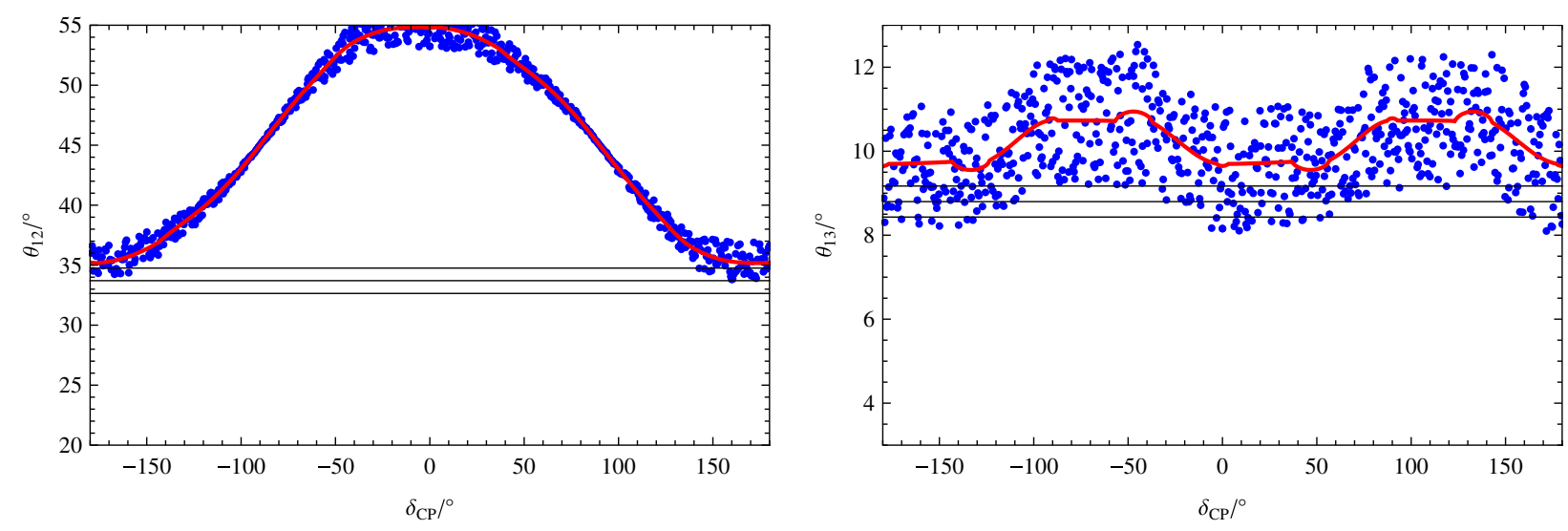

FIG. 1. Predictions on the PMNS mixing angles. Shown are the dependencies of the angles $\theta_{12}$ (left panel) and $\theta_{13}$ (right panel) on $\delta_{C P}$. Red lines correspond to the charged fermion masses fixed at the central values according to the RGE running in Ref. [53]; blue points are masses randomly generated within $1 \sigma$ allowed regions. The black lines show the best-fit values and $1 \sigma$ bounds of $\theta_{13}$ and $\theta_{12}$ from Ref. [62]. We take $\sin \theta_{C}=0.225$.

$U_{\mathrm{PMNS}}=\left(\begin{array}{ccc}\frac{1}{2}\left(\tilde{s}_{l}^{*}+\sqrt{2} c_{l}\right) & -\frac{\tilde{s}_{l}^{*}}{2}+\frac{c_{l}}{\sqrt{2}} & \frac{\tilde{s}_{l}^{*}}{\sqrt{2}} \\ \frac{\tilde{s}_{l}}{\sqrt{2}}-\frac{c_{l}}{2} & \frac{1}{2}\left(\sqrt{2} \tilde{s}_{l}+c_{l}\right) & -\frac{c_{l}}{\sqrt{2}} \\ -\frac{1}{2} & \frac{1}{2} & \frac{1}{\sqrt{2}}\end{array}\right)$.

Notice that the matrix of phases $P_{l}$ does not appear here since it can be removed by the rephasing of the fields. From Eq. (62), one finds mixing parameters in the standard parametrization,

$$
\begin{gathered}
s_{13}=\frac{s_{l}}{\sqrt{2}}, \\
s_{12}^{2}=\frac{1}{2}-\frac{\sqrt{2} c_{l} s_{l} \cos \phi_{l}}{2-s_{l}^{2}}, \\
s_{23}^{2}=\frac{c_{l}^{2}}{2-s_{l}^{2}} \approx \frac{1}{2}\left(1-\frac{1}{2} s_{l}^{2}\right),
\end{gathered}
$$

$$
\sin \delta_{\mathrm{CP}}=-\sin \phi_{l}-s_{l}^{2} \sin \phi_{l} \cos ^{2} \phi_{l}+\mathcal{O}\left(s_{l}^{3}\right) .
$$

The above results can be expressed in terms of known variables $\left(\theta_{C}, m_{s}, m_{d}, m_{\mu}, m_{e}\right)$ and $\phi_{l}$. Although $\phi_{l} \approx \phi_{C}$, we cannot connect it to the $C P$ violation in the CKM mixing without introducing 1-3 and 2-3 mixing. Using expression (C18) for the mixing parameter $s_{l}$ and replacing $\phi_{l}$ by $\delta_{\mathrm{CP}}$, we obtain from Eq. (63)

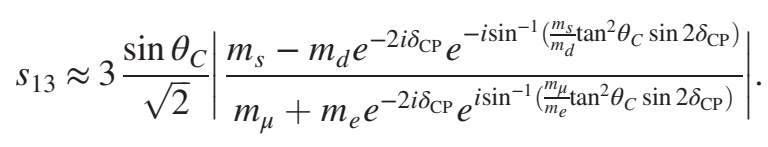

From Eqs. (63) and (64), we obtain the relation between observables:

$$
s_{12}^{2} \approx \frac{1}{2}+\frac{s_{13} \cos \delta_{\mathrm{CP}}}{c_{13}^{2}}
$$

In Fig. 1 , we show the mixing angles $\theta_{12}$ and $\theta_{13}$ as functions of the $C P$ phase $\delta_{\mathrm{CP}} \approx \phi_{l}$ according to Eqs. (64) and (63). We used $\sin \theta_{C}=0.225$ and the values of $m_{s}, m_{d}$, $m_{\mu}$, and $m_{e}$ from Ref. [53]. The angle $\theta_{23}$ is in the first octant: $\sin ^{2} \theta_{23}=0.49$. Taking the $1 \sigma$ allowed interval for $\theta_{12}$, we obtain from (68) $\cos \delta_{C P}<-0.86$ or

$$
\delta_{C P} \in(0.80 \pi, 1.16 \pi)
$$

Including 2-3 mixing in $V_{\mathrm{CKM}} \simeq U_{l}$ changes the prediction for $\delta_{\mathrm{CP}}$ by a few degrees. The result (69) is in agreement with general phenomenological analyses [59-61] for the case of $\mathrm{BM}$ mixing receiving corrections from charge lepton mixing $U_{l}$. According to Ref. [61], $\cos \delta_{\mathrm{CP}}$ should be in the range $[-1.00,-0.72]$ at $3 \sigma$ confidence level.

The upper value of the interval (69) is in agreement with global fit results at about $1 \sigma$ level. Notice that $\delta_{C P}$ is strongly restricted here by the requirement of maximal possible reduction of the 1-2 mixing from its $\mathrm{BM}$ value $\sin ^{2} \theta_{12}=0.5$. So, the best value would be $\cos \delta_{C P}=-1$. The only other parameter that enters the relation (68) is the 1-3 mixing, which is measured very precisely. If we would use the TBM matrix $U_{0}=U_{\text {TBM }}$ instead of BM, no large corrections from $U_{l}^{\dagger}$ are required, and $\cos \delta_{C P}$ should be close to zero. However, $U_{0}=U_{\mathrm{TBM}}$ cannot be obtained in our residual symmetry approach. Thus, future measurements of $\delta_{C P}$ will test the scenario.

The values of mixing angles are subjects of the renormalization group (RG) corrections. The CKM mixing receives small corrections. For the PMNS mixing in our framework, the RG corrections can be significant due to strong hierarchy of the right-handed neutrino masses. The strong hierarchy originates from the up-type quark-mass spectrum, as the 
right-handed neutrino mass matrix is determined by $m_{D}^{T} m_{\nu}^{-1} m_{D}$, and in $\mathrm{SO}(10)$ models, $m_{D}$ is the same as $M_{u}$ (or approximately the same in multi-Higgs variations). From this, one obtains the masses of the order $\left(10^{4}, 10^{9}\right.$, $\left.10^{14}\right) \mathrm{GeV}$. Between the lightest and heaviest right-handed neutrinos, one or two of them are integrated out, while the others remain in the RG equations. This is where RG running may have larger effects on the flavor structure [63].

The RG effects with such a strong hierarchy have been studied in Ref. [18]. In the standard model (SM) extended by RH neutrinos, the corrections mainly depend on the lightest neutrino mass, Majorana phases, and the mass ordering. The result in Ref. [18] shows that for $m_{1}=$ $10^{-3} \mathrm{eV}$ in the case of normal mass ordering the correction to $\theta_{12}$ is in the range $\left(-1.5^{\circ}, 1.0^{\circ}\right)$. For smaller/larger $m_{1}$, the correction can be significantly suppressed/enhanced. For example, when $m_{1}=10^{-4} \mathrm{eV}$, the correction can be reduced down to $0.02^{\circ}$ (cf. Fig. 10 in Ref. [18]), independent of the Majorana phases, while for $m_{1}=10^{-2} \mathrm{eV}$ with zero Majorana phases, it can reach $10^{\circ}$.

The other two mixing angles $\theta_{13}$ and $\theta_{23}$ are generally much more stable with respect to the RG corrections than $\theta_{12}$ [64-66]. In the scenario with strong hierarchy of righthanded neutrino masses, the correction to the 1-3 mixing is found to be always smaller than $0.3^{\circ}$ in the SM, which is negligible compared to the uncertainties caused by the fermion masses at the GUT scale-see Eq. (60).

The fermion singlets $S$ from the hidden sector may produce further corrections. Since the mass scale of $S$ is not much higher than the GUT scale, we expect small RG corrections from, e.g., $10^{16}$ to $10^{18} \mathrm{GeV}$. At the GUT scale or below, the singlets can be integrated out, generating the heavy Majorana masses of right-handed neutrinos. Therefore, we can assume that the SM plus type-I seesaw is valid up to the GUT scale, and in this range, one can adopt the conclusions from Ref. [18]. Above the GUT scale, up to the mass scale of $S$, we expect small RG corrections due to the small interval or running. Furthermore, the mixing of these singlets with active neutrinos is strongly suppressed.

Variations of the $C P$ phase predictions may be possible. Essentially, the result (69) is obtained in the assumption of negligible RG corrections. Large RG corrections $\left(\sim 10^{\circ}\right)$ to the 1-2 mixing can be obtained for the degenerate pair of $\nu_{1}$ and $\nu_{2}$. This can be realized for $m_{1} \sim 10^{-2} \mathrm{eV}$ or invertedmass ordering. In this case, RG corrections can reduce $\theta_{12}$ down to $35^{\circ}$, and large corrections from $U_{l}$ are not needed. Consequently, $\cos \delta_{C P}$ can be small.

One can use some other matrices from Table I, e.g., $U_{G R}$. This, however, would require large corrections from $U_{l}^{\dagger}$ and its substantial deviation from $V_{\mathrm{CKM}}$. Another possibility is to produce some mixing from the portal interactions. Finally, one can abandon the residual symmetry approach and fix structure of $M_{S}$ using other symmetries or principles.
Finally, let us comment on the viability of leptogenesis in this framework. As previously mentioned, the typical $\mathrm{RH}$ neutrino masses are $\left(10^{4}, 10^{9}, 10^{14}\right) \mathrm{GeV}$, which is very hierarchical. The lightest RH neutrino in this scenario is too light to produce the observed baryon asymmetry [67]. However, the second RH neutrino is in the proper mass range and may realize successful $N_{2}$ leptogenesis (see, e.g., Refs. [68,69]).

\section{CONCLUSIONS}

The relation between the lepton and quark mixings [cf. Eq. (1)] can imply the grand unification and existence of the hidden sector, which is connected to the visible sector via the RH neutrino portal. The grand unification ensures the approximate equality $U_{l} \approx V_{d} \approx V_{\mathrm{CKM}}$ and, consequently, $U_{\mathrm{PMNS}} \simeq V_{\mathrm{CKM}}^{\dagger} U_{0}$, whereas the hidden sector with certain symmetries generates $U_{0}$ and produces the smallness of neutrino masses.

We focus on the symmetry aspects of this scenario-the interplay of discrete flavor symmetries and the $\mathrm{SO}(10)$ gauge symmetry. We develop the residual symmetry approach to generate $U_{0}$, which connects the visible and hidden sectors. The $\mathbb{Z}_{2} \times \mathbb{Z}_{2}$ residual symmetries of the visible and hidden sectors are intrinsic symmetries of the SO(10) Yukawa interactions.

Embedding of these residual symmetries into a unified finite flavor group fixes the moduli of mixing matrix elements in the form of cosines of rational multiples of $\pi$. Imposing the unitarity condition results in only a few forms of $U_{0}$ that include the BM matrix. Using these matrices, we reconstruct the group presentations and thus identified the corresponding symmetry groups. The flavor symmetry is broken in the hidden sector spontaneously. In the visible sector (at lower energy scales), the breaking can be explicit. In the latter case, only the basis symmetry in the hidden sector is promoted to a larger non-Abelian symmetry. We consider a specific model that realizes the BM mixing for $U_{0}$.

We use the 126-plet with the Planck-scale mass or composite 126-plet originating from the Plank-scale physics to generate the CKM mixing and $U_{l} \sim V_{\mathrm{CKM}}$ as well as the differences of quark and lepton masses of the second and the first generations. Thus, generation of CKM mixing and the mass differences of the down quarks and charge leptons of the first the second generations are connected. Interestingly, the CKM mixing and the corresponding $U_{l}$ matrix can be reproduced with an additional contribution from the Planck-scale physics with the flavor structure similar to the one for $S$ and, consequently, the light neutrinos.

Assuming that the RGE corrections are small, we expect the leptonic $C P$ violation phase to be in the range $144^{\circ} \lesssim \delta_{\mathrm{CP}} \lesssim 210^{\circ}$. Future measurements of $\delta_{\mathrm{CP}}$ in accelerator neutrino experiments such as $\mathrm{T} 2 \mathrm{~K}$, NOVA, and DUNE will be an important test of this scenario. 
Coupling of the hidden sector with the visible one realizes the double-seesaw mechanism. It allows one to disentangle the generation of the $U_{0}$ mixing and the CKM mixing. The latter is related to the Planck scale-suppressed nonrenormalizable interactions.

An important feature of this scenario is a very strong hierarchy of masses of the RH neutrinos. This can lead to significant renormalization group effects that correct, in particular, the 1-2 mixing. The lightest RH neutrino with mass $10^{4} \mathrm{GeV}$ has mixing with active neutrinos of the order $10^{-7}$. So, it cannot be observed at colliders but could play some role in leptogenesis. The $N_{2}$ leptogenesis can be realized.

Future precise measurements of the $C P$ phase, establishing mass ordering and absolute scale (degeneracy) of masses, will provide important tests of the scenario. In particular, establishing a strong normal mass hierarchy and substantial $C P$ violation would exclude the simplest realization based on the BM mixing from the hidden sector.

No new physics related to the neutrino-mass generation should be observed at LHC and other future collider experiments. Proton decay might be detected at some level.

\section{ACKNOWLEDGMENTS}

X. J. X. would like to thank Patrick Ludl, who has done an early work in this framework. The author appreciates many discussions with him, both on physics and industry.

\section{APPENDIX A: SYMMETRY GROUP CONDITION}

All the $T$ 's and $R$ 's in Eqs. (14) and (15) are SU(3) matrices, i.e., $\operatorname{det} T=\operatorname{det} R=1$, which implies that the product $T_{i} R_{j}$ is also an $\mathrm{SU}(3)$ matrix. One can use properties of $\mathrm{SU}(3)$ matrices to derive the relations below.

Introducing the eigenvalues of $W_{i j} \equiv T_{i} R_{j}\left(\lambda_{1}, \lambda_{2}, \lambda_{3}\right)$, we can represent this matrix as

$$
W_{i j}=T_{i} R_{j}=U_{T S}\left(\begin{array}{lll}
\lambda_{1} & & \\
& \lambda_{2} & \\
& & \lambda_{3}
\end{array}\right) U_{T S}^{\dagger} .
$$

Then, according to Eq. (16), $\lambda_{i}^{p}=1$. Keeping in mind that the eigenvalues satisfy the relations $\left|\lambda_{1}\right|^{2}=\left|\lambda_{2}\right|^{2}=\left|\lambda_{3}\right|^{2}=$ $\lambda_{1} \lambda_{2} \lambda_{3}=1$ (the latter follows from $\operatorname{det} W_{i j}=1$ ), we can parametrize them as

$$
\lambda_{1}=e^{-i\left(\theta_{2}+\theta_{3}\right)}, \quad \lambda_{2}=e^{i \theta_{2}}, \quad \lambda_{3}=e^{i \theta_{3}}
$$

with

$$
\theta_{i}=\frac{2 \pi n_{i}}{p}
$$

Since unitary transformations do not change the trace of a matrix, we have

$$
\operatorname{tr}\left(T_{i} R_{j}\right)=\sum_{i} \lambda_{i}=e^{-i\left(\theta_{2}+\theta_{3}\right)}+e^{i \theta_{2}}+e^{i \theta_{3}} .
$$

For a given $p$, this sum has discrete sets of values.

On the other hand, we compute the trace of $T_{i} R_{j}$ from Eqs. (14) and (15),

$$
\operatorname{tr}\left(T_{i} R_{j}\right)=4\left(U_{0}\right)_{i j}\left(U_{0}\right)_{i j}^{*}-1=4\left|\left(U_{0}\right)_{i j}\right|^{2}-1,
$$

which is a real number. Therefore, Eq. (A4) must be a real number, too, which requires that

$$
\sin \theta_{2}+\sin \theta_{3}-\sin \left(\theta_{2}+\theta_{3}\right)=0 .
$$

This equation has only three solutions in the range $[0,2 \pi)$,

$$
\theta_{2}=0, \quad \theta_{3}=0, \quad \theta_{2}+\theta_{3}=0
$$

or, equivalently, $n_{1}=0, n_{2}=0, n_{3}=0$. Consequently, in any of these three cases, the eigenvalues of $T_{i} R_{j}$ can be taken as $\left(1, e^{2 \pi n / p}, e^{-2 \pi n / p}\right)$, and thus the trace equals

$$
\operatorname{tr}\left(T_{i} R_{j}\right)=1+2 \cos \frac{2 \pi n_{i j}}{p_{i j}}=4 \cos ^{2} \frac{\pi n_{i j}}{p_{i j}}-1 .
$$

Using Eqs. (A5) and (A8), we immediately obtain equality (17).

\section{APPENDIX B: THE $S_{4}$ GROUP AND ITS REPRESENTATIONS}

The group $S_{4}$ is the permutation group of four objects. It can be defined by four generators $r_{1}, r_{2}, t_{1}$, and $t_{2}$ with the following relations:

$$
\begin{gathered}
r_{1}^{2}=r_{2}^{2}=t_{1}^{2}=t_{2}^{2}=1, \\
\left(t_{1} r_{1}\right)^{4}=\left(t_{1} r_{2}\right)^{4}=\left(t_{2} r_{1}\right)^{3}=\left(t_{2} r_{2}\right)^{3}=1 .
\end{gathered}
$$

The group has five irreducible representations, denoted as $\mathbf{1}, \mathbf{1}^{\prime}, \mathbf{2}, \mathbf{3}$, and $\mathbf{3}^{\prime}$, in which the generators are represented by the matrices

$$
\begin{aligned}
& R_{1}^{(\mathbf{3})}=-\frac{1}{2}\left(\begin{array}{ccc}
0 & \sqrt{2} & \sqrt{2} \\
\sqrt{2} & 1 & -1 \\
\sqrt{2} & -1 & 1
\end{array}\right), \quad R_{2}^{(\mathbf{3})}=-\left(\begin{array}{ccc}
1 & 0 & 0 \\
0 & 0 & 1 \\
0 & 1 & 0
\end{array}\right), \\
& T_{1}^{(\mathbf{3})}=\left(\begin{array}{ccc}
1 & 0 & 0 \\
0 & -1 & 0 \\
0 & 0 & -1
\end{array}\right), \quad T_{2}^{(\mathbf{3})}=\left(\begin{array}{ccc}
-1 & 0 & 0 \\
0 & 1 & 0 \\
0 & 0 & -1
\end{array}\right) ;
\end{aligned}
$$




$$
\begin{aligned}
& R_{1}^{\left(\mathbf{3}^{\prime}\right)}=\left(\begin{array}{ccc}
0 & 1 & 0 \\
1 & 0 & 0 \\
0 & 0 & 1
\end{array}\right), \quad R_{2}^{\left(\mathbf{3}^{\prime}\right)}=\left(\begin{array}{ccc}
-1 & 0 & 0 \\
0 & -1 & 0 \\
0 & 0 & 1
\end{array}\right), \quad T_{1}^{\left(\mathbf{3}^{\prime}\right)}=\left(\begin{array}{ccc}
1 & 0 & 0 \\
0 & -1 & 0 \\
0 & 0 & -1
\end{array}\right), \quad T_{2}^{\left(\mathbf{3}^{\prime}\right)}=\left(\begin{array}{ccc}
1 & 0 & 0 \\
0 & 0 & 1 \\
0 & 1 & 0
\end{array}\right) ; \\
& R_{1}^{(2)}=\left(\begin{array}{cc}
-1 & 0 \\
0 & 1
\end{array}\right), \quad R_{2}^{(2)}=\left(\begin{array}{cc}
1 & 0 \\
0 & 1
\end{array}\right), \quad T_{1}^{(\mathbf{2})}=\left(\begin{array}{cc}
1 & 0 \\
0 & 1
\end{array}\right), \quad T_{2}^{(\mathbf{2})}=\left(\begin{array}{cc}
\frac{1}{2} & \frac{\sqrt{3}}{2} e^{-\frac{\pi i}{6}} \\
\frac{\sqrt{3}}{2} e^{\frac{\pi i}{6}} & -\frac{1}{2}
\end{array}\right)
\end{aligned}
$$

where $\omega \equiv \exp \left(\frac{2 \pi i}{3}\right)$, and

$$
\begin{array}{cccc}
R_{1}^{\left(\mathbf{1}^{\prime}\right)}=-1, & R_{2}^{\left(\mathbf{1}^{\prime}\right)}=1, & T_{1}^{\left(\mathbf{1}^{\prime}\right)}=1, & T_{2}^{\left(\mathbf{1}^{\prime}\right)}=-1 ; \\
R_{1}^{(\mathbf{1})}=1, & R_{2}^{(\mathbf{1})}=1, & T_{1}^{(\mathbf{1})}=1, & T_{2}^{(\mathbf{1})}=1 .
\end{array}
$$

The Clebsch-Gordan coefficients are given by

$$
\begin{aligned}
& \left(\begin{array}{l}
x_{1} \\
x_{2} \\
x_{3}
\end{array}\right)^{(3)} \otimes\left(\begin{array}{l}
y_{1} \\
y_{2} \\
y_{3}
\end{array}\right)^{(\mathbf{3})}=\left(\frac{x_{1} y_{1}+x_{2} y_{2}+x_{3} y_{3}}{\sqrt{3}}\right)^{(\mathbf{1})} \oplus \frac{1}{6 \sqrt{2}}\left(\begin{array}{c}
6 x_{1} y_{1}-3\left(x_{2}+x_{3}\right)\left(y_{2}+y_{3}\right) \\
e^{\frac{\pi i}{6} \sqrt{3}}\left(2 x_{1} y_{1}-x_{2}\left(y_{2}-3 y_{3}\right)+x_{3}\left(3 y_{2}-y_{3}\right)\right)
\end{array}\right)^{(\mathbf{2})} \\
& \oplus \frac{1}{\sqrt{2}}\left(\begin{array}{c}
x_{3} y_{2}-x_{2} y_{3} \\
x_{1} y_{3}-x_{3} y_{1} \\
x_{2} y_{1}-x_{1} y_{2}
\end{array}\right)^{(3)} \oplus \frac{1}{2}\left(\begin{array}{c}
\sqrt{2}\left(x_{2} y_{2}-x_{3} y_{3}\right) \\
x_{2} y_{1}-x_{3} y_{1}+x_{1}\left(y_{2}-y_{3}\right) \\
-\left(x_{2} y_{1}+x_{3} y_{1}+x_{1}\left(y_{2}+y_{3}\right)\right)
\end{array}\right)^{\left(3^{\prime}\right)} \\
& \left(\begin{array}{l}
x_{1} \\
x_{2} \\
x_{3}
\end{array}\right)^{\left(\mathbf{3}^{\prime}\right)} \otimes\left(\begin{array}{l}
y_{1} \\
y_{2} \\
y_{3}
\end{array}\right)^{\left(\mathbf{3}^{\prime}\right)}=\left(\frac{x_{1} y_{1}+x_{2} y_{2}+x_{3} y_{3}}{\sqrt{3}}\right)^{(\mathbf{1})} \oplus \frac{1}{\sqrt{2}}\left(\begin{array}{c}
x_{1} y_{1}-x_{2} y_{2} \\
\frac{e^{\frac{\pi i}{6}}\left(x_{1} y_{1}+x_{2} y_{2}-2 x_{3} y_{3}\right)}{\sqrt{3}}
\end{array}\right)^{(2)} \\
& \oplus \frac{1}{2}\left(\begin{array}{c}
\sqrt{2}\left(-x_{3} y_{2}+x_{2} y_{3}\right) \\
\left(x_{2} y_{1}+x_{3} y_{1}-x_{1}\left(y_{2}+y_{3}\right)\right) \\
\left(-x_{2} y_{1}+x_{3} y_{1}+x_{1}\left(y_{2}-y_{3}\right)\right)
\end{array}\right)^{(3)} \oplus \frac{1}{\sqrt{2}}\left(\begin{array}{c}
\left(x_{3} y_{2}+x_{2} y_{3}\right) \\
\left(x_{3} y_{1}+x_{1} y_{3}\right) \\
\left(x_{2} y_{1}+x_{1} y_{2}\right)
\end{array}\right)^{\left(3^{\prime}\right)}, \\
& \left(\begin{array}{l}
x_{1} \\
x_{2}
\end{array}\right)^{(\mathbf{2})} \otimes\left(\begin{array}{l}
y_{1} \\
y_{2}
\end{array}\right)^{(\mathbf{2})}=\left(\frac{x_{1} y_{1}-\omega x_{2} y_{2}}{\sqrt{2}}\right)^{(\mathbf{1})} \oplus\left(\frac{x_{1} y_{2}-x_{2} y_{1}}{\sqrt{2}}\right)^{\left(\mathbf{1}^{\prime}\right)} \oplus \frac{1}{\sqrt{2}}\left(\begin{array}{c}
x_{2} y_{1}+x_{1} y_{2} \\
-\omega^{2} x_{1} y_{1}-x_{2} y_{2}
\end{array}\right)^{\left(\mathbf{2}^{\prime}\right)} \text {. }
\end{aligned}
$$

\section{APPENDIX C: ANALYTIC DIAGONALIZATION IN THE VISIBLE SECTOR}

To diagonalize the mass matrices in Eq. (48), we reconstruct the mass matrices of down quarks and charged leptons in terms of mass eigenstates and mixing angles, $M_{d}=U_{d} \operatorname{diag}\left(\tilde{m}_{d}, \tilde{m}_{s}, \tilde{m}_{b}\right) U_{d}^{T}$ and $M_{l}=U_{l} \operatorname{diag}\left(\tilde{m}_{e}, \tilde{m}_{\mu}, \tilde{m}_{\tau}\right) U_{l}^{T}$, which gives

$$
\begin{aligned}
& \left(\begin{array}{cc}
\tilde{m}_{d} c^{2}+\tilde{s}^{2} \tilde{m}_{s} & c\left(\tilde{s} \tilde{m}_{s}-\tilde{m}_{d} \tilde{s}^{*}\right) \\
c\left(\tilde{s} \tilde{m}_{s}-\tilde{m}_{d} \tilde{s}^{*}\right) & \tilde{m}_{s} c^{2}+\tilde{m}_{d} \tilde{s}^{* 2}
\end{array}\right)=\left(\begin{array}{cc}
d_{1}+k_{1} & f e^{i \phi_{f}} \\
f e^{i \phi_{f}} & d_{2}+k_{2}
\end{array}\right), \\
& \left(\begin{array}{cc}
\tilde{m}_{e} c_{e}^{2}+\tilde{s}_{e}^{2} \tilde{m}_{\mu} & c_{e}\left(\tilde{s}_{e} \tilde{m}_{\mu}-\tilde{m}_{e} \tilde{s}_{e}^{*}\right) \\
c_{e}\left(\tilde{s}_{e} \tilde{m}_{\mu}-\tilde{m}_{e} \tilde{s}_{e}^{*}\right) & \tilde{m}_{\mu} c_{e}^{2}+\tilde{m}_{e} \tilde{s}_{e}^{* 2}
\end{array}\right)=\left(\begin{array}{cc}
-3 d_{1}+k_{1} & -3 f e^{i \phi_{f}} \\
-3 f e^{i \phi_{f}} & -3 d_{2}+k_{2}
\end{array}\right) .
\end{aligned}
$$

Because $\tilde{m}_{d} \tilde{s}^{* 2} \ll \tilde{m}_{s} c^{2}$ and $\tilde{m}_{e} \tilde{s}_{e}^{* 2} \ll \tilde{m}_{\mu} c_{e}^{2}$, we neglect $\tilde{m}_{d} \tilde{s}^{* 2}$ and $\tilde{m}_{e} \tilde{s}_{e}^{* 2}$ below. Equating the corresponding elements of the matrices on the left-hand side and right-hand side, we obtain expressions for $d_{1,2}$ and $k_{1,2}$ : 


$$
\begin{aligned}
& k_{1}=\frac{1}{4}\left(3 c^{2} \tilde{m}_{d}+c_{l}^{2} \tilde{m}_{e}+\tilde{m}_{\mu} \tilde{s}_{l}^{2}+3 \tilde{m}_{s} \tilde{s}^{2}\right), \\
& k_{2}= \frac{1}{4}\left(3 c^{2} \tilde{m}_{s}+c_{l}^{2} \tilde{m}_{\mu}\right), \\
& d_{1}=\frac{1}{4}\left(c^{2} \tilde{m}_{d}-c_{l}^{2} \tilde{m}_{e}-\tilde{m}_{\mu} \tilde{s}_{l}^{2}+\tilde{m}_{s} \tilde{s}^{2}\right), \\
& d_{2}=\frac{1}{4}\left(c^{2} \tilde{m}_{s}-c_{l}^{2} \tilde{m}_{\mu}\right) .
\end{aligned}
$$

Since $-3 d_{2}+k_{2}$ and $d_{2}+k_{2}$ in Eqs. (C2) and (C1) are real under our assumptions, $\tilde{m}_{s}$ and $\tilde{m}_{\mu}$ should be approximately real, either positive or negative. Because $d_{2}$ is dominant, we take positive $\tilde{m}_{s}$ and negative $\tilde{m}_{\mu}$, i.e., $\tilde{m}_{s} \approx m_{s}$ and $\tilde{m}_{\mu} \approx-m_{\mu}$. Furthermore, the equalities $\operatorname{Im}\left(d_{1}+k_{1}\right)=$ $\operatorname{Im}\left(-3 d_{1}+k_{1}\right)=0$ give

$$
\begin{aligned}
& c^{2} m_{d} \sin \alpha_{d}+s^{2} m_{s} \sin 2 \phi_{C} \\
& \quad=c_{e}^{2} m_{e} \sin \alpha_{e}-s_{l}^{2} m_{\mu} \sin 2 \phi_{l}=0,
\end{aligned}
$$

which leads to

$\sin \alpha_{d}=-\frac{s^{2} m_{s}}{c^{2} m_{d}} \sin 2 \phi_{C}, \quad \sin \alpha_{e}=\frac{s_{l}^{2} m_{\mu}}{c_{l}^{2} m_{e}} \sin 2 \phi_{l}$

From the equality of the off-diagonal elements of Eqs. (C1) and (C2), we obtain

$$
f e^{i \phi_{f}}=c\left(\tilde{s} m_{s}-\tilde{m}_{d} \tilde{s}^{*}\right)=\frac{1}{3} c_{l}\left(\tilde{s}_{l} m_{\mu}+\tilde{m}_{e} \tilde{s}_{e}^{*}\right)
$$

or

$$
\begin{aligned}
2 f e^{i \phi_{f}} & =\sin 2 \theta_{C}\left(m_{s} e^{i \phi_{C}}-\tilde{m}_{d} e^{-i \phi_{C}}\right) \\
& =\frac{1}{3} \sin 2 \theta_{e}\left(m_{\mu} e^{i \phi_{l}}+\tilde{m}_{e} e^{-i \phi_{l}}\right) .
\end{aligned}
$$

Because $m_{s} \gg m_{d}$ and $m_{\mu} \gg m_{e}$, one can immediately see from Eq. (C8) that the phases $\phi_{C}$ and $\phi_{l}$ should be approximately equal to $\phi_{f}$ :

$$
\phi_{C}=\phi_{f}+\mathcal{O}\left(\frac{m_{d}}{m_{s}}\right), \quad \phi_{l}=\phi_{f}+\mathcal{O}\left(\frac{m_{e}}{m_{\mu}}\right) .
$$

This reproduces the result in Eq. (56). Equation (C8) also gives

$$
\frac{\sin 2 \theta_{l}}{\sin 2 \theta_{C}}=\frac{3\left(m_{s} e^{i \phi_{C}}-\tilde{m}_{d} e^{-i \phi_{C}}\right)}{m_{\mu} e^{i \phi_{e}}+\tilde{m}_{e} e^{-i \phi_{l}}} \approx \frac{3\left(m_{s}+m_{d} e^{i \phi_{1}}\right)}{m_{\mu}+m_{e} e^{i \phi_{2}}},
$$

$$
\phi_{1} \equiv \pi+\alpha_{d}-2 \phi_{f}, \quad \phi_{2} \equiv \alpha_{e}-2 \phi_{f},
$$

where we have taken the approximation $\phi_{C} \approx \phi_{f} \approx \phi_{e}$.

Because of the relations $m_{s} \gg m_{d}$ and $m_{\mu} \gg m_{e}$, in Eq. (C10), the imaginary parts in $m_{d} e^{i \phi_{1}}$ and $m_{e} e^{i \phi_{2}}$ can be neglected, which leads to Eq. (57).

Finally, we express $d_{1,2}, k_{1,2}$, and $f$ in terms of $\left(m_{d}, m_{s}, m_{e}, m_{\mu}, \theta_{C}, \phi_{l}\right)$ and $\theta_{l}$ inserting results of Eqs. (C9) and (C6) into Eqs. (C3), (C4), and (C7):

$$
\begin{aligned}
k_{1}= & \frac{1}{4}\left(3 \sqrt{c^{4} m_{d}^{2}-s^{4} m_{s}^{2} \sin ^{2} 2 \phi_{l}}\right. \\
& +\sqrt{c_{l}^{4} m_{e}^{2}-s_{l}^{4} m_{\mu}^{2} \sin ^{2}\left(2 \phi_{l}\right)} \\
& \left.+\cos 2 \phi_{l}\left(3 s^{2} m_{s}-s_{e}^{2} m_{\mu}\right)\right),
\end{aligned}
$$

$$
\begin{aligned}
d_{1}= & \frac{1}{4}\left(\sqrt{c^{4} m_{d}^{2}-s^{4} m_{s}^{2} \sin ^{2} 2 \phi_{l}}\right. \\
& \left.-\sqrt{c_{l}^{4} m_{e}^{2}-s_{l}^{4} m_{\mu}^{2} \sin ^{2} 2 \phi_{l}}+\cos 2 \phi_{l}\left(s_{l}^{2} m_{\mu}+s^{2} m_{s}\right)\right),
\end{aligned}
$$

$$
k_{2}=\frac{1}{4}\left(3 c^{2} m_{s}-c_{l}^{2} m_{\mu}\right),
$$

$$
d_{2}=\frac{1}{4}\left(c^{2} m_{s}+c_{l}^{2} m_{\mu}\right)
$$

$$
f e^{i \phi_{f}}=s c\left[m_{s} e^{i \phi_{C}}-m_{d} \exp \left(-i \alpha_{d}-i \phi_{C}\right)\right]
$$

Here,

$$
\alpha_{d}=-\arcsin \left(\frac{s^{2} m_{s}}{c^{2} m_{d}} \sin 2 \phi_{C}\right)
$$

The lepton mixing can be obtained from (C10)

$\sin 2 \theta_{l} \approx 3 \sin 2 \theta_{C}\left|\frac{m_{s}-e^{-2 i \phi_{l}} e^{-i \sin ^{-1}\left(\frac{s^{2} \sin \left(2 \phi_{l}\right) m_{s}}{c^{2} m_{d}}\right)} m_{d}}{m_{\mu}+e^{-2 i \phi_{l}} e^{i \sin ^{-1}\left(\frac{\sin \left(2 \phi_{l}\right) m_{\mu} s_{l}^{2}}{c_{e}^{2} m_{e}}\right)} m_{e}}\right|$,

where the right-hand side still contains $\theta_{l}$ but it only appears in the negligibly small term proportional to $m_{e}$. So, one can simply replace $\theta_{l}$ by $\theta_{C}$ in this term.

We have checked that our analytic results agree with numerical computations up to an order of $10^{-4}$. 
[1] C. Giunti and M. Tanimoto, Phys. Rev. D 66, 053013 (2002).

[2] M. Raidal, Phys. Rev. Lett. 93, 161801 (2004).

[3] H. Minakata and A. Yu. Smirnov, Phys. Rev. D 70, 073009 (2004).

[4] P. H. Frampton and R. N. Mohapatra, J. High Energy Phys. 01 (2005) 025.

[5] J. Ferrandis and S. Pakvasa, Phys. Rev. D 71, 033004 (2005).

[6] F. Vissani, arXiv:hep-ph/9708483.

[7] V. D. Barger, S. Pakvasa, T. J. Weiler, and K. Whisnant, Phys. Lett. B 437, 107 (1998).

[8] P. F. Harrison, D. H. Perkins, and W. G. Scott, Phys. Lett. B 530, 167 (2002).

[9] P. F. Harrison and W. G. Scott, Phys. Lett. B 535, 163 (2002).

[10] I. Esteban, M. C. Gonzalez-Garcia, M. Maltoni, I. MartinezSoler, and T. Schwetz, J. High Energy Phys. 01 (2017) 087.

[11] K. Cheung, S. K. Kang, C. S. Kim, and J. Lee, Phys. Rev. D 72, 036003 (2005).

[12] Z.-Z. Xing, Phys. Lett. B 618, 141 (2005).

[13] A. Datta, L. Everett, and P. Ramond, Phys. Lett. B 620, 42 (2005).

[14] S. Antusch and S. F. King, Phys. Lett. B 631, 42 (2005).

[15] J. Harada, Europhys. Lett. 75, 248 (2006).

[16] S. Antusch, S. F. King, and R. N. Mohapatra, Phys. Lett. B 618, 150 (2005).

[17] N. Li and B.-Q. Ma, Phys. Rev. D 71, 097301 (2005).

[18] M. A. Schmidt and A. Yu. Smirnov, Phys. Rev. D 74, 113003 (2006).

[19] K. A. Hochmuth and W. Rodejohann, Phys. Rev. D 75, 073001 (2007).

[20] F. Plentinger, G. Seidl, and W. Winter, Phys. Rev. D 76, 113003 (2007).

[21] Y. Zhang, X. Zhang, and B.-Q. Ma, Phys. Rev. D 86, 093019 (2012).

[22] X. Zhang, Y.-j. Zheng, and B.-Q. Ma, Phys. Rev. D 85, 097301 (2012).

[23] J. Harada, Europhys. Lett. 103, 21001 (2013).

[24] H.-W. Ke, T. Liu, and X.-Q. Li, Phys. Rev. D 90, 053009 (2014).

[25] G. Sharma and B. C. Chauhan, J. High Energy Phys. 07 (2016) 075.

[26] G. Sharma, S. Bhardwaj, B. C. Chauhan, and S. Verma, arXiv:1711.08796.

[27] P. Minkowski, Phys. Lett. 67B, 421 (1977).

[28] T. Yanagida, KEK Report No. 79-18, 1979.

[29] M. Gell-Mann, P. Ramond, and R. Slansky, Conf. Proc. C790927, 315 (1979).

[30] S. Glashow, Study Inst. Ser. B Phys. 59, 687 (1979).

[31] R. Mohapatra and G. Senjanovic, Phys. Rev. Lett. 44, 912 (1980).

[32] R. N. Mohapatra and J. W. F. Valle, Phys. Rev. D 34, 1642 (1986).

[33] M. Lindner, M. A. Schmidt, and A. Yu. Smirnov, J. High Energy Phys. 07 (2005) 048.

[34] C. Hagedorn, M. A. Schmidt, and A. Yu. Smirnov, Phys. Rev. D 79, 036002 (2009).

[35] H. Georgi, AIP Conf. Proc. 23, 575 (1975).
[36] H. Fritzsch and P. Minkowski, Ann. Phys. (N.Y.) 93, 193 (1975).

[37] P. O. Ludl and A. Yu. Smirnov, Phys. Rev. D 92, 073010 (2015).

[38] X. Chu and A. Yu. Smirnov, J. High Energy Phys. 05 (2016) 135.

[39] B. Bajc and A. Yu. Smirnov, Nucl. Phys. B909, 954 (2016).

[40] R. N. Mohapatra, Phys. Rev. Lett. 56, 561 (1986).

[41] C. S. Lam, Phys. Lett. B 656, 193 (2007).

[42] C. S. Lam, Phys. Rev. Lett. 101, 121602 (2008).

[43] C. S. Lam, Phys. Rev. D 78, 073015 (2008).

[44] C. S. Lam, Phys. Rev. D 89, 095017 (2014).

[45] R. N. Mohapatra and S. Nussinov, Phys. Rev. D 60, 013002 (1999).

[46] Y. Nomura and T. Yanagida, Phys. Rev. D 59, 017303 (1998).

[47] P. H. Frampton, S. T. Petcov, and W. Rodejohann, Nucl. Phys. B687, 31 (2004).

[48] G. Altarelli, F. Feruglio, and L. Merlo, J. High Energy Phys. 05 (2009) 020.

[49] H.-J. He and X.-J. Xu, Phys. Rev. D 86, 111301 (2012).

[50] GAP Group, GAP-Groups, Algorithms, and Programming, Ver 4.8.8, 2017.

[51] R. M. Fonseca and W. Grimus, J. High Energy Phys. 09 (2014) 033.

[52] P. S. Bhupal Dev, B. Dutta, R. N. Mohapatra, and M. Severson, Phys. Rev. D 86, 035002 (2012).

[53] C. R. Das and M. K. Parida, Eur. Phys. J. C 20, 121 (2001).

[54] R. de Adelhart Toorop, F. Feruglio, and C. Hagedorn, Phys. Lett. B 703, 447 (2011).

[55] R. de Adelhart Toorop, F. Feruglio, and C. Hagedorn, Nucl. Phys. B858, 437 (2012).

[56] W. Rodejohann and X.-J. Xu, Phys. Rev. D 91, 056004 (2015).

[57] G. Senjanovic, in Proceedings, International Conference, SEESAW25 (2005), p. 45.

[58] K. S. Babu, J. C. Pati, and F. Wilczek, Nucl. Phys. B566, 33 (2000).

[59] I. Girardi, S. T. Petcov, and A. V. Titov, Nucl. Phys. B894, 733 (2015).

[60] I. Girardi, S. T. Petcov, and A. V. Titov, Eur. Phys. J. C 75, 345 (2015).

[61] S. T. Petcov, arXiv:1711.10806.

[62] C. Patrignani et al. (Particle Data Group Collaboration), Chin. Phys. C 40, 100001 (2016).

[63] S. Antusch, J. Kersten, M. Lindner, M. Ratz, and M. A. Schmidt, J. High Energy Phys. 03 (2005) 024.

[64] S. Antusch, J. Kersten, M. Lindner, and M. Ratz, Nucl. Phys. B674, 401 (2003).

[65] W. Rodejohann and X.-J. Xu, Nucl. Phys. B899, 463 (2015).

[66] W. Rodejohann and X.-J. Xu, Phys. Rev. D 96, 055039 (2017).

[67] S. Davidson and A. Ibarra, Phys. Lett. B 535, 25 (2002).

[68] P. Di Bari and M. Re Fiorentin, J. Cosmol. Astropart. Phys. 03 (2016) 039.

[69] P. Di Bari and S. F. King, J. Cosmol. Astropart. Phys. 10 (2015) 008. 